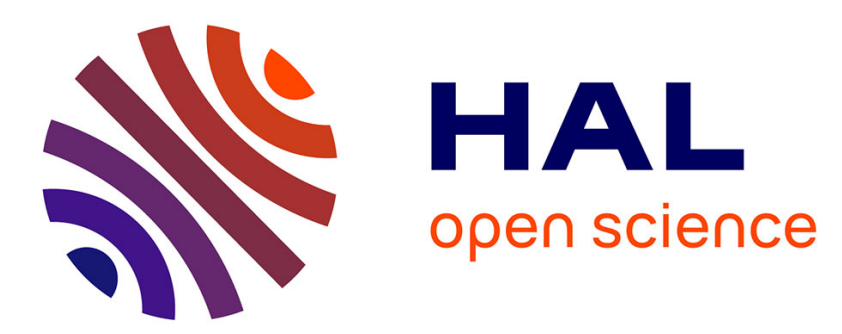

\title{
Invertible and non-invertible information sets in linear rational expectations models
}

\author{
Brad Baxter, Liam Graham, Stephen Wright
}

\section{To cite this version:}

Brad Baxter, Liam Graham, Stephen Wright. Invertible and non-invertible information sets in linear rational expectations models. Journal of Economic Dynamics and Control, 2010, 35 (3), pp.295. 10.1016/j.jedc.2010.11.002 . hal-00767497

\section{HAL Id: hal-00767497 \\ https://hal.science/hal-00767497}

Submitted on 20 Dec 2012

HAL is a multi-disciplinary open access archive for the deposit and dissemination of scientific research documents, whether they are published or not. The documents may come from teaching and research institutions in France or abroad, or from public or private research centers.
L'archive ouverte pluridisciplinaire HAL, est destinée au dépôt et à la diffusion de documents scientifiques de niveau recherche, publiés ou non, émanant des établissements d'enseignement et de recherche français ou étrangers, des laboratoires publics ou privés. 


\section{Author's Accepted Manuscript}

Invertible and non-invertible information sets in linear rational expectations models

Brad Baxter, Liam Graham, Stephen Wright

PII: $\quad$ S0165-1889(10)00248-4

DOI: $\quad$ doi:10.1016/j.jedc.2010.11.002

Reference: $\quad$ DYNCON 2501

To appear in: $\quad$ Journal of Economic Dynamics

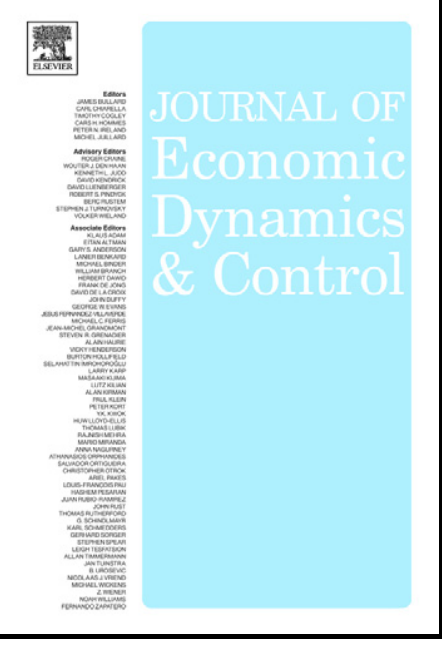

www.elsevier.com/locate/jedc \& Control

Received date: 15 August 2008

Accepted date: 2 November 2010

Cite this article as: Brad Baxter, Liam Graham and Stephen Wright, Invertible and noninvertible information sets in linear rational expectations models, Journal of Economic Dynamics \& Control, doi:10.1016/j.jedc.2010.11.002

This is a PDF file of an unedited manuscript that has been accepted for publication. As a service to our customers we are providing this early version of the manuscript. The manuscript will undergo copyediting, typesetting, and review of the resulting galley proof before it is published in its final citable form. Please note that during the production process errors may be discovered which could affect the content, and all legal disclaimers that apply to the journal pertain. 


\title{
Invertible and non-invertible information sets in linear rational expectations models*
}

\author{
Brad Baxter $†$ Liam Graham ${ }^{\ddagger}$ and Stephen Wright ${ }^{\S}$
}

November 10, 2010

\begin{abstract}
Rational expectations solutions are usually derived by assuming that all state variables relevant to forward-looking behaviour are directly observable, or that they are "...an invertible function of observables" (Mehra and Prescott, 1980). Using a framework that nests linearised DSGE models, we give a number of results useful for the analysis of linear rational expectations models with restricted information sets. We distinguish between instantaneous and asymptotic invertibility, and show that the latter may require significantly less information than the former. We also show that non-invertibility of the information set can have significant implications for the time-series properties of economies.
\end{abstract}

JEL classifications: D80; E32.

Keywords: imperfect information; invertibility; rational expectations; fundamental versus nonfundamental time series representations; Kalman filter; dynamic stochastic general equilibrium

*We thank Michel Juillard (the Editor) and two anonymous referees for their comments which have greatly improved the paper. We have also benefited from discussions with Paul Levine, Marco Lippi, Roland Meeks, Kristoffer Nimark and Joseph Pearlman.

${ }^{\dagger}$ School of Economics, Maths \& Statistics Birkbeck College, University of London.

$\ddagger$ Corresponding author: Department of Economics, University College London, Gower Street, London WC1E 6BT, UK. Liam.Graham@ucl.ac.uk. Phone: +44 207679 5850. Fax: +44 207916 2775 .

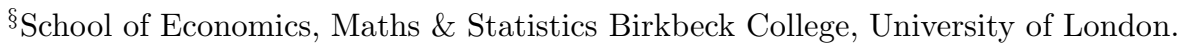




\section{Introduction}

Underlying most rational expectations models is the strong assumption that all state variables relevant to forward-looking behaviour are directly observable. This can be rationalized, as in Mehra and Prescott (1980) by the assumption that the states are "...an invertible function of observables".

In this paper we consider a general linear rational expectations framework that nests linearised dynamic stochastic general equilibrium (DSGE) models, in which the Kalman filter is used to estimate the state variables from a given information set. Compared to standard applications of the Kalman filter, the signal extraction problem is complicated by the responses of forward-looking variables to new information feeding back both on the measurement problem and on future values of the state variables. Within this framework we analyse the implications of three kinds of information sets:

1. Instantaneously invertible information sets. The states can be recovered from only $t$ - dated information, the Kalman filter is redundant and the full information solution is replicated.

2. Asymptotically invertible information sets. The economy converges to a solution in which the Kalman filter replicates full information. We show that the conditions for asymptotic invertibility can be satisfied with fewer observables than needed for instantaneous invertibility. However this puts important additional restrictions on the nature of the measurement process and its interaction with the dynamics of the underlying states.

3. Non-invertible information sets. The Kalman filter can never replicate the full information solution. We show that the impact of limited information is transitory, but can be highly persistent and that the endogenous nature of the filtering problem introduces new (but unobservable) sources of dynamics in response to structural shocks. The only observable dynamics will be those of the estimated, as opposed to the actual states. We show that, with non-invertible information sets, the estimated states will follow the same vector autoregressive process as would the true states in a notional full information economy, but with a different covariance pattern of shocks. In this notional economy, pre-determined variables like capital are subject to "pseudo-shocks" that are logically impossible under full information.

We also examine the implications of information for the time series properties of the economy. Invertibility of an information set is closely related to invertibility or fundamentalness in time series analysis. The solution to a Kalman filter problem always results in a fundamental time series representation of the observables i.e. one in which the innovations can be recovered from the history of the observables. 
But invertibility of the information set implies that the structural innovations that drive the true states are also fundamental in the time series sense. In contrast, under non-invertible information sets the structural innovations cannot be recovered from the history of the observables. Indeed, in the special case of a non-invertible information set in which the number of observables equals the number of structural innovations, we show that the latter are also (up to a scaling factor) innovations to a nonfundamental time series representation of the observables. We also show that in general the endogenous nature of the filtering problem leads to nonbasic (Lippi and Reichlin, 1994) nonfundamental representations of the observables.

To complement the paper we provide a Matlab toolkit which allows the easy application of our techniques to a wide class of linear rational expectations models.

Our analysis builds on past analysis of macroeconomic models with limited information (Pearlman et al., 1986; Pearlman, 1992; Svensson and Woodford, 2003, 2004). These papers also examine signal extraction problems in the context of both forms of endogeneity noted above and we show that our solution is identical to theirs. However, by showing that the solution to the endogenous Kalman filter problem can be expressed in terms of the solution to a "parallel problem" in which the states and the measurement process are fully exogenous, we are able to exploit known results in control theory (Anderson and Moore, 1979) to show the conditions under which the Kalman filter converges to a unique steady state. This in turn allows us to derive the conditions under which an information set is asymptotic invertible. We also show that our analysis of time series properties is closely related to the literature on econometric inference in time series representations (Hansen and Sargent, 2005; Lippi and Reichlin, 1994; Fernandez-Villaverde et al., 2007).

Limited information sets within macroeconomic models may arise as in Svensson and Woodford (2003) and Pearlman (1992), where a policymaker sets policy variables with incomplete information on the underlying state variables in the economy, or as in Bomfim (2004), Keen (2010), Collard and Dellas (2010) where representative consumers are assumed to face informational restrictions, or state variables are measured with error. Sims (2003) shows in a model of "rational inattention", in which agents face information processing capacity constraints, that the optimal response to these constraints can be represented as a signal extraction problem of the same form. Woodford (2003), Lorenzoni (2009), Nimark (2007), Graham and Wright (2010) and Porapakkarm and Young (2008) address the problem of heterogeneous agents facing a symmetric filtering problem of inferring aggregates which requires them to form estimates of a "hierarchy of average expectations" (Townsend, 1983). While this is a more complex problem, Graham and Wright (2010) show 
that when such models include endogenous states a filtering problem of the form discussed in the present paper is central to the solution.

The remainder of the paper is organized as follows. Section 2 summarises the key properties of the general filtering problem within a general linear rational expectations model. In Section 3 we give conditions for asymptotic invertibility and in Section 4 we discuss non-invertible information sets. Section 5 discusses implications for time series properties. In Section 6 we show how our techniques can be applied to an analytical example based on the benchmark stochastic growth model. Appendices, available online, provide proofs and derivations.

\section{The signal extraction problem}

\subsection{A general system representation}

A structural linear rational expectations model can be written as in McCallum (1998):

$$
\begin{aligned}
A_{y y} E_{t} y_{t+1} & =B_{y y} y_{t}+B_{y k} k_{t}+B_{y z} z_{t} \in \mathbb{R}^{q \times 1} \\
k_{t+1} & =B_{k y} y_{t}+B_{k k} k_{t}+B_{k z} z_{t} \in \mathbb{R}^{r_{k} \times 1} \\
z_{t+1} & =B_{z z} z_{t}+\zeta_{t+1} \in \mathbb{R}^{r_{z} \times 1}
\end{aligned}
$$

with $\left\{k_{0}, z_{0}\right\}$ given. In the first block of equations $y_{t}$ is a $q \times 1$ vector of nonpredetermined variables. The matrix $A_{y y}$ may not be invertible. The equations in this first block may be first-order conditions (both static and dynamic), identities or production relations. The second block describes the evolution of an $r_{k} \times 1$ vector of predetermined variables, $k_{t}$, while the third describes the evolution of an $r_{z} \times 1$ vector of exogenous stochastic processes, $z_{t}$, that can be represented by a first order vector autoregression, with $\zeta_{t}$ an $r_{z} \times 1$ vector of iid innovations.

Throughout the paper we give examples taken from linearised DSGE models, however the model of (1) to (3) is more general than this.

We assume expectations are formed based on an information set

$$
I_{t}=\left\{\left\{i_{j}\right\}_{j=0}^{t} ; \Xi ; \Phi_{0}\right\}
$$

where $\Xi$ contains the (time-invariant) structure and parameters of equations (1) to (4); $\Phi_{0}$ represents initial estimates of the states and their covariance matrix ${ }^{1}$ and $i_{t}$

\footnotetext{
${ }^{1}$ One value for $\Phi_{0}$ would be the unconditional properties of the states implicit in $\Xi$. Under conditions given in Proposition 2, initial estimates will have no impact on the equilibrium.
} 
is an $n \times 1$ vector of observed variables

$$
i_{t}=C_{i k} k_{t}+C_{i z} z_{t}+C_{i y} y_{t}+C_{i w} w_{t} \in \mathbb{R}^{n \times 1}
$$

where $w_{t}$ is an $r_{w} \times 1$ vector of measurement errors, with $0 \leq r_{w} \leq n .^{2}$ For generality we can in principle allow these to be serially correlated by representing them as a vector autoregression:

$$
w_{t+1}=B_{w w} w_{t}+\omega_{t+1} \in \mathbb{R}^{r_{w} \times 1}
$$

where $\omega_{t}$ is a vector of iid innovations.

The nature of the information set (which will be model-specific) should ideally be explicitly related to the underlying structure of the economy. ${ }^{3}$ It should also be borne in mind that, as we show in Appendix A, the structure of the model in (1) to (3) needs to be both informationally consistent and informationally feasible i.e. any linear combinations of the states appearing in the forward looking equations of (1) must be observable.

We provide a Matlab toolkit which takes as input a system in the form specified in equations (1) to (6) and implements all the transformations and solution methods that follow. ${ }^{4}$

\subsection{The filtering problem}

For compactness of notation we incorporate predetermined and exogenous variables, $k_{t}$ and $z_{t}$, together with the measurement errors, $w_{t}$, into a vector of state variables $\xi_{t}$ of dimension $r=r_{k}+r_{z}+r_{w}$. We partition $y_{t}$ into two sub-vectors, $c_{t} \in \mathbb{R}^{m \times 1}$, with $m \leq q$, containing observable forward-looking variables that satisfy expectational difference equations such as consumption or policy variables and $x_{t} \in \mathbb{R}^{(q-m) \times 1}$ containing variables given by static relationships. None of our results depend on this partition. 5

In Appendix A we show that we can then use (2) to (5) to derive the following compact representation of the state evolution and measurement equations

$$
\xi_{t+1}=F_{\xi} \xi_{t}+F_{c} c_{t}+v_{t+1} \in \mathbb{R}^{r \times 1}
$$

\footnotetext{
${ }^{2}$ Measurement errors may be of lower dimension than the measured variables themselves if, for example, some linear combination of $k_{t}, z_{t}$ and $y_{t}$ is measured without error, or if measurement errors in different variables are systematically related.

${ }^{3}$ For example, Graham and Wright (2010) argue that, in an incomplete markets setting, information should be assumed to be "market-consistent" - i.e. agents only obtain information from the markets they trade in. Porapakkarm and Young (2008) is another example of this approach.

${ }^{4}$ Available from the authors' websites.

${ }^{5}$ This is discussed in more detail in Appendix A.
} 


$$
i_{t}=H_{\xi}^{\prime} \xi_{t}+H_{c} c_{t} \in \mathbb{R}^{n \times 1}
$$

where $F_{\xi} \in \mathbb{R}^{r \times r} ; F_{c} \in \mathbb{R}^{r \times m} ; c_{t} \in \mathbb{R}^{m \times 1}, H_{\xi} \in \mathbb{R}^{r \times n} ; H_{c} \in \mathbb{R}^{n \times m}, \xi_{0}$ is given and

$$
\begin{aligned}
\xi_{t} & =\left[\begin{array}{c}
k_{t} \\
z_{t} \\
w_{t}
\end{array}\right] \in \mathbb{R}^{r \times 1} ; v_{t}=F_{u} u_{t} \in \mathbb{R}^{r \times 1} \\
u_{t} & =\left[\begin{array}{c}
\zeta_{t} \\
\omega_{t}
\end{array}\right] \in \mathbb{R}^{s \times 1} ; F_{u}=\left[\begin{array}{cc}
0_{r_{k} \times r_{k}} & 0_{r_{k} \times s} \\
0_{s \times r_{k}} & I_{s}
\end{array}\right] \in \mathbb{R}^{r \times s} \\
S & \equiv E\left(u_{t} u_{t}^{\prime}\right) \in \mathbb{R}^{s \times s} ; Q=E\left(v_{t} v_{t}^{\prime}\right)=F_{u} S F_{u}^{\prime} \in \mathbb{R}^{r \times r}
\end{aligned}
$$

where we assume that the vector of underlying structural disturbances $u_{t}$ is jointly iid with covariance matrix $S$ and we assume $\operatorname{rank}(S)=\operatorname{rank}(Q)=s \equiv r_{z}+r_{w}$; $\operatorname{rank}\left(H_{\xi}\right)=n$. Definitions of the matrices in (7) and (8) are given in Appendix A.

The signal extraction problem is solved using the Kalman filter. The standard Kalman filter (as in e.g. Harvey, 1981, 1989; Hamilton, 1994; Hansen and Sargent, 2005) can be written as a special case of our system:

$$
\begin{aligned}
\xi_{t+1} & =F_{\xi} \xi_{t}+v_{t+1} \\
i_{t} & =H_{\xi}^{\prime} \xi_{t}+w_{t}
\end{aligned}
$$

Comparing this with the general system in (7) and (8) reveals a number of important differences.

First, there are two forms of endogeneity of the system in (7) and (8) to the solution to the signal extraction problem. If $F_{c} \neq 0$ there is "dynamic endogeneity", for example in the stochastic growth model capital depends on lagged consumption. If $H_{c} \neq 0$ there is "measurement endogeneity", for example via intratemporal optimality conditions. ${ }^{6}$ Second, when the $\xi_{t}$ are exogenous it is typically assumed that the eigenvalues of the matrix $F_{\xi}$ in (12) are not greater than unity in absolute value. In contrast, in the problem generated by a typical DSGE model, $F_{\xi}$ will have at least one explosive eigenvalue, due to the dynamics of capital under dynamic efficiency. ${ }^{7}$ We shall show that this feature interacts in interesting ways with the endogeneity of the Kalman filter. Third, whereas in the standard representation (12) there are typically innovations to each of the states, if $\xi_{t}$ in the general representation contains pre-determined variables, the $r \times 1$ vector of innovations $v_{t}$ will have zeros in

\footnotetext{
${ }^{6}$ Endogeneity of this form makes the filtering problem in the context of a DSGE much closer to those analysed in control theory, as in e.g. Whittle (1983) Anderson and Moore (1979) and Söderstörm (1994).

${ }^{7}$ The model in Section 6 has this property.
} 
its first $r_{k}$ rows. Hence the true structural innovations $u_{t}$, defined in (10), are only of dimension $s=r-r_{k} \leq r$. Fourth (and more trivially) the measurement errors, $w_{t}$, have been absorbed into the redefined states, $\xi_{t} \cdot{ }^{8}$

In the remainder of the paper the only features of the system in (7) and (8) that are crucial to our results are the overall state dimension, $r$, the "stochastic dimension", $s \leq r$ and the number of measured variables, $n \leq r$, along with the endogeneity of both states and measured variables to the forward-looking variables, $c_{t}$.

\subsection{Full information solution}

The special case of full information provides a crucial analytical building block for the more general solution under other information sets.

Definition 1 (Full information) Full information implies the state variables are known, $i_{t}=\xi_{t}$.

Full information is a special case of the system (7) and (8) with $n=r, H_{\xi}=$ $I_{r}, H_{c}=0$. The Kalman filter is therefore redundant.

The solution for the forward-looking variables under full information that satisfies the expectational difference equations in (1) can then be expressed in the form

$$
c_{t}^{*}=\eta^{\prime} \xi_{t}^{*}
$$

where for any variable $x_{t}, x_{t}^{*}$ denotes its value under full information. The matrix $\eta \in \mathbb{R}^{r x m}$, depends in general on all structural and preference parameters of the model and can be computed using standard techniques (e.g. Blanchard and Kahn, 1980 ; McCallum, 1998). ${ }^{9}$ For the rest of the paper we assume it to be unique and treat it as parametric. Given (12), the full information states follow a first order vector autoregressive process in reduced form:

$$
\xi_{t+1}^{*}=G \xi_{t}^{*}+v_{t+1}
$$

where

$$
G=F_{\xi}+F_{c} \eta^{\prime} \in \mathbb{R}^{r x r}
$$

\footnotetext{
${ }^{8}$ This allows us to accommodate both serial correlation of measurement errors $w_{t}$, and contemporaneous correlation with the innovations, $\zeta_{t}$ to the exogenous variables $z_{t}$; it also simplifies the key formulae.

${ }^{9}$ Note that all elements in the $i$ th row of $\eta$ are zero for $i>r_{k}+r_{z}$ (measurement errors have no impact on $c_{t}$ under full information).
} 
The behaviour of the forward-looking variables, $c_{t}$, under full information turns out to be crucial for the stability of the states under any information set. As noted in the previous section, in a model with endogenous capital $F_{\xi}$ will usually have at least one explosive eigenvalue. This latent explosive property can only be controlled by the behaviour of the forward-looking variables. Under full information this stabilisation follows directly from the standard rational expectations solution. Under standard conditions the matrices $\eta$ and $F_{c}$ always satisfy the following conditions:

Assumption 1. All the eigenvalues of the matrix $G=F_{\xi}+F_{c} \eta^{\prime}$ have real parts less than or equal to unity

Assumption 2. Let $G=V \Lambda V^{-1}$ where $\Lambda$ is a diagonal matrix of eigenvalues and $V$ the corresponding matrix of eigenvectors. For any strictly unit eigenvalue in $\Lambda$ the corresponding row of $F_{c}$ is zero.

Assumption 1 rules out explosive rational expectations solutions; ${ }^{10}$ Assumption 2 states that, to the extent that any innovations have permanent effects, these are innovations to strictly exogenous processes (e.g. there may be a unit root component in technology). ${ }^{11}$ These features of the solution under full information turn out to be equally crucial for the stability of the solution even when the information set is non-invertible and thus does not replicate full information.

\subsection{Instantaneous invertibility}

The full information solution is replicated under the following conditions:

Definition 2 (Instantaneous invertibility). An information set is instantaneously invertible if $n=r$, the number of observables is equal to the state dimension and $H_{\xi}$ in the measurement equation (8) is invertible. Hence full information can be replicated using only $t$ - dated information.

In this case the state variables can be obtained by inverting the measurement equation (8) to give

$$
\xi_{t}=\left(H_{\xi}^{\prime}\right)^{-1}\left(i_{t}-H_{c} c_{t}\right)
$$

hence again the Kalman filter is redundant, as is all lagged information on the observables, $\left\{i_{j}\right\}_{j=0}^{t-1}$.

\footnotetext{
${ }^{10}$ Note that there is no direct link between the explosive eigenvalues in the rational expectations solution of (1) to (3) (which are set to zero in solving the model and deriving $\eta$ under full information), and those of $F_{\xi}$, except to the extent that, by inspection of (14), the stability of the rational expectations solution requires that the explosive eigenvalues of $F_{\xi}$ be "switched off" by the behaviour of the forward-looking variables.

${ }^{11}$ Assumption 2 follows naturally from the underlying structural model in (1) to (3), since the dependence of state variables on $c_{t}$ is only via $k_{t+1}$, hence all elements of the $i$ th row of $F_{c}$ are zero for $i>r_{k} \equiv r-s$.
} 


\subsection{The Kalman filter}

For the remainder of the paper we shall assume that the information set is not instantaneously invertible. We therefore need to apply the Kalman filter.

Given the linear structure of the model we follow standard practice ${ }^{12}$ and conjecture that the solutions for the forward-looking variables $c_{t}$ will be "certaintyequivalent" i.e.

$$
c_{t}=\eta^{\prime} \widehat{\xi}_{t}
$$

where $\widehat{\xi}_{t}=E \xi_{t} \mid I_{t}$ is the optimal estimate of the current state vector given the available information set $I_{t}$ and $\eta$ is identical to the matrix for the full information case in (12). We show below that this conjecture is verified.

We first define two key matrices that characterise the properties of the state estimates and state forecasts.

$$
M_{t}=E\left[\left(\xi_{t}-\widehat{\xi}_{t}\right)\left(\xi_{t}-\widehat{\xi}_{t}\right)^{\prime}\right] \in \mathbb{R}^{r \times r}
$$

is the covariance matrix of the filtering errors of the state estimates and

$$
P_{t+1}=E\left[\left(\xi_{t+1}-E_{t} \xi_{t+1}\right)\left(\xi_{t+1}-E_{t} \xi_{t+1}\right)^{\prime}\right] \in \mathbb{R}^{r \times r}
$$

is the covariance matrix of the one-step ahead state forecast errors. ${ }^{13}$

The following proposition shows that despite the endogeneity of both states and measured variables to the forward-looking variables, the solution to the filtering problem can be related to standard results:

Proposition 1 (The parallel problem). In the solution to the "endogenous" Kalman filter problem given by (7) and (8), the mean squared error matrices $M_{t}$ and $P_{t+1}$ in (17) and (18) are identical to those derived from a parallel exogenous Kalman filter problem

$$
\begin{aligned}
\widetilde{\xi}_{t+1} & =F_{\xi} \widetilde{\xi}_{t}+v_{t+1} \\
\widetilde{i}_{t} & =H_{\xi}^{\prime} \widetilde{\xi}_{t}
\end{aligned}
$$

(i.e. setting $F_{c}=0$ and $H_{c}=0$ in (7) and (8)) so are given by the standard Kalman

\footnotetext{
${ }^{12}$ See, for example, Pearlman (1992); Svensson and Woodford (2004), who in turn are simply applying standard results in control theory as in e.g. Whittle (1983) and Söderström (1994).

${ }^{13}$ For compactness of notation we write the period $t$ estimate of the states as $\widehat{\xi}_{t}$; whereas the standard Kalman filter literature commonly uses $\widehat{\xi}_{t \mid t}$. For the forecast at time $t$ of the states at period $t+1$ we write $E_{t} \xi_{t+1}\left(=E_{t} \widehat{\xi}_{t+1}\right)$ instead of the standard $\widehat{\xi}_{t+1 \mid t} . P_{t+1}$ is commonly denoted $P_{t+1 \mid t}$, and using the same notation $M_{t}=P_{t \mid t}$, but we separate the two for clarity.
} 
filter recursion

$$
\begin{aligned}
P_{t+1} & =F_{\xi} M_{t} F_{\xi}^{\prime}+Q \\
M_{t} & =\left(I_{r}-\widetilde{\beta}_{t} H_{\xi}^{\prime}\right) P_{t} \\
\widetilde{\beta}_{t} & =P_{t} H_{\xi}\left(H_{\xi}^{\prime} P_{t} H_{\xi}\right)^{-1} \in \mathbb{R}^{r \times n}
\end{aligned}
$$

where $Q=E\left(v_{t} v_{t}^{\prime}\right)$ is as defined in (11). In the solution to the actual problem, (i.e. with $F_{c} \neq 0, H_{c} \neq 0$ and $c_{t}$ given by (16)), the estimated states follow the process

$$
\begin{gathered}
\widehat{\xi}_{t+1}=G \widehat{\xi}_{t}+\beta_{t} \varepsilon_{t+1} \\
\beta_{t}=\widetilde{\beta}_{t}\left(I_{n}+H_{c} \eta^{\prime} \widetilde{\beta}_{t}\right)^{-1}
\end{gathered}
$$

where $G$ is as defined in (14) and $\varepsilon_{t}$ is the vector of innovation to the observables,

$$
\varepsilon_{t+1} \equiv i_{t+1}-E_{t} i_{t+1}
$$

Proof. See Appendix B.

The filtering problem set out in (7) and (8) displays two forms of endogeneity. Proposition 1 states that the solution to this problem can be derived from the solution to a parallel filtering problem for a notional state process $\widetilde{\xi}$ and a notional set of measured variables $\widetilde{i}_{t}$ in which there is no endogeneity, so that the standard exogenous Kalman filter formulae can be applied. ${ }^{14}$ This is the case because, while the forward-looking variables determine future states in the true problem via the matrix $F_{c}$, this does not impact on one-step ahead uncertainty (since the marginal impact of today's choices on tomorrow's states is known today even if current states are unknown). As a result the expression for $P_{t+1}$ only allows for the direct impact of uncertainty about today's states transmitting to uncertainty about tomorrow's states, via the matrix $F_{\xi}$. Since the matrix $F_{c}$ does not affect the solution to the filtering problem, it can be solved under the assumption that $F_{c}=0$.

The Kalman gain ${ }^{15}$ matrix $\beta_{t}$ for the true problem is not however the same as its

\footnotetext{
${ }^{14}$ Our formulae for $P_{t+1}$ and $\widetilde{\beta}_{t}$ are more compact than the more common formulation, given our absorption of measurement error into the states, but can be easily shown to be identical.

${ }^{15}$ We use the definition of the Kalman gain as in Harvey (1981), in which it can be interpreted as a matrix of regression coefficients updating current state estimates in response to forecast errors in predicting measured variables (Söderström, 1994, refers to this as the "filter gain") . The term is also frequently applied (as in Hamilton, 1994) to a matrix, often denoted $K$, that updates forecasts of the states in response to the same forecast errors (Söderström, 1994 refers to this definition as the "predictor gain"). In the parallel exogenous problem $\widetilde{K}=F_{\xi} \widetilde{\beta}_{t}$ in our notation, however in the actual endogenous problem $K=G \beta_{t}$, since it would incorporate the endogenous response of forward-looking variables both in $\beta_{t}$ but also in the autoregressive representation in (22).
} 
counterpart $\widetilde{\beta}_{t}$ in the parallel problem because the signal conveyed by innovations to the measured variables also affects the forward-looking variables $c_{t}$. But this has no impact on the mean squared error matrices $M_{t}$ and $P_{t+1}$, hence these can be derived under the assumption that $H_{c}=0$.

Proposition 1 shows that, conditional upon the solution for $\beta_{t}$ in (23), the estimated states $\widehat{\xi}_{t}$ follow the process given by (22) with the same non-explosive autoregressive matrix $G$ as for the true states under full information, in (13). In the parallel problem, in contrast, the notional state process $\widetilde{\xi}_{t}$ has autoregressive ma$\operatorname{trix} F_{\xi}$, which, as noted above may have explosive eigenvalues. We shall see below that this rather unusual feature of the state process in the parallel problem implies interesting interactions between the signal extraction problem and time series properties.

In Appendix B we show that the solution to the filtering problem given by Proposition 1 is implied by standard results in control theory (see Söderström, 1994, Ch. 6); it is also equivalent to that in Pearlman et al. (1986) and Svensson and Woodford (2003). ${ }^{16}$ However the specification in terms of the parallel problem has, as far as we know, gone unremarked and it is this feature that provides the basis for many of the results that follow.

\subsection{The steady state Kalman filter}

Equations (19) to (21) are a set of recursive matrix equations, for which it is natural to look for a stable steady state. The re-statement of the endogenous Kalman filter problem in Proposition 1 in terms of the parallel exogenous problem means that this becomes a straightforward application of existing results.

Proposition 2 (steady-state Kalman filter). If the parallel problem in Proposition 1 is stabilisable and detectable in the sense of Anderson and Moore (1979), then, as $t \rightarrow \infty$ the endogenous Kalman filter converges to a unique steady state satisfying (19) to (21): $P_{t} \rightarrow P, M_{t} \rightarrow M$ and $\beta_{t} \rightarrow \beta$ for any initial positive definite matrix $P_{0}$.

Proof. See Appendix C.

The twin conditions of stabilisability and detectability can both be related to the nature of the structural innovations that drive the system in (7) and (8). The

\footnotetext{
${ }^{16}$ In Appendix B we show that Svensson and Woodford's derivation requires that state estimates update in response to the forecast errors in the parallel, rather than the true forecast errors, but that their assumed Kalman gain matrix is consistent with this assumption, so that their solution is the same as ours.
} 
two conditions can be written as

$$
\begin{array}{rll}
\text { stabilisability: } & \left|\Lambda_{i}\left(F_{\xi}+F_{u} L_{1}^{\prime}\right)\right|<1 \quad \forall i \\
\text { detectability: } & \left|\Lambda_{i}\left(F_{\xi}+L_{2} H_{\xi}^{\prime}\right)\right|<1 \quad \forall i
\end{array}
$$

for some matrices $L_{1} \in \mathbb{R}^{r \times s}$ and $L_{2} \in \mathbb{R}^{r \times n}$, where $F_{u}$ is as defined in (10) and $\Lambda_{i}(A)$ denotes the $i$ th eigenvalue of a matrix $A$. Note that these conditions apply to the "parallel problem" of Proposition 1 and hence are entirely unaffected by endogeneity in the true filtering problem.

The first condition, stabilisability, is trivial if there are no pre-determined variables $\left(r_{k}=0\right)$ and hence $u_{t}$ the structural innovations defined in (10) are of dimension $s=r$, since in that case $F_{u}=I_{r}$. Where there are pre-determined variables $(s<r)$ it is not so straightforward. As noted above, $F_{\xi}$ may have at least one explosive eigenvalue and in this case $F_{u}$ will contain a row of zeros in exactly the row corresponding to an explosive eigenvalue in $F_{\xi}$, so that the condition for stabilisability can only be met if the relevant row of $F_{\xi}$ contains off-diagonal elements. A simple example might be that capital must depend not only on lagged consumption, but also on lags of stochastic exogenous state variables (for example technology). While this does put restrictions on the underlying structure of the model we have not encountered examples of DSGE models where the condition for stabilisability is not satisfied.

The second condition, detectability, requires that there must be some observable indicator, however poor, of any state variables with associated explosive or unit eigenvalues.

It is common practice to solve the model using steady-state values of $\beta, P$ and $M$. However, while the recursion in equations (19) to (21) does not depend on the data it does depend on the passage of time. Hence the use of steady-state values makes the implicit assumption that there is a sufficiently long history of $i_{t}$ in the information set for the Kalman filter to have converged.

\section{$3 \quad$ Asymptotic invertibility}

We have derived the Kalman filter for a general signal extraction problem where we have assumed only that the information set is not instantaneously invertible as in Definition 2 (if it were, as we saw in Section 2.4, the Kalman filter would be redundant). In general the information set will be non-invertible: i.e. state estimates will differ from the true states. However, under certain conditions on the 
nature of the information set we now show that the Kalman filter will converge to a steady state that replicates full information.

Definition 3 (asymptotic invertibility) A sequence of information sets $\left\{I_{j}\right\}_{j=1}^{t}$ is asymptotically invertible if

$$
M \equiv \lim _{t \rightarrow \infty} M_{t}\left(I_{t}\right)=0
$$

where $M_{t}=E\left(\xi_{t}-\widehat{\xi}_{t}\right)\left(\xi_{t}-\widehat{\xi}_{t}\right)^{\prime}$ and, from Proposition 2, $M$ satisfies the steady state of the recursion in (20).

Definition 3 explicitly notes the dependence of $M_{t}$ on $I_{t}$ and hence on the entire history of observables. This contrasts with instantaneous invertibility, which, from Definition 2 requires only $t$-dated information. Instantaneous invertibility also requires that $n$, the number of measured variables, equals $r$, the number of states. In most standard exogenous Kalman filter problems, in which the stochastic dimension, $s$, equals the state dimension, $r$, this is the only way that full information can be replicated. However, the endogenous nature of the states in in the context of DSGE models implies that this condition is sufficient, but not necessary.

In DSGE models, the dynamic endogeneity of some of the states means that, because they are pre-determined, the stochastic dimension $s$ is less than $r$, the state dimension. There may as a result be cases in which the Kalman filter converges to a steady state that replicates full information. While this is less demanding in terms of the number of observables required to replicate full information, it does require significant restrictions on the nature of the information set and its relation to the underlying structural model. These are summarised in the following proposition:

Proposition 3 (conditions for asymptotic invertibility). Assume that the Kalman filter of Proposition 1 satisfies the conditions in Proposition 2. A sequence of information sets $\left\{I_{j}\right\}_{j=1}^{t}$ is asymptotically invertible if and only if

1. $n=s$

2. $H_{\xi}^{\prime} F_{u}$ is invertible

3. $\left|\Lambda_{i}\left(\left(I-\widetilde{\beta}(Q) H_{\xi}^{\prime}\right) F_{\xi}\right)\right|<1 \quad \forall i$

where $n$ is the number of observed variables; $s=\operatorname{rank}(S)=\operatorname{rank}(Q)$ is the stochastic dimension of the state variables; $H_{\xi}$ is as given in equation (8); S, $Q$ and $F_{u}$ are as defined in (11) and (10); $\Lambda_{i}(A)$ are the eigenvalues of a matrix $A$; and $\widetilde{\beta}(Q)$ $=F_{u}\left(H_{\xi}^{\prime} F_{u}\right)^{-1}$ satisfies (21) setting $P=Q$. 
Proof. See Appendix E.

To illustrate, assume that all three conditions in Proposition 3 hold and that there is a sufficiently long history of the observables that state estimates in period $t$ have converged on their true values. Since all the conditions relate to features of the parallel problem as defined in Proposition 1 we can ignore both forms of endogeneity and set $F_{c}=H_{c}=0 .{ }^{17}$ Manipulation of equations (7), (8), (10) then implies that in period $t+1$, the innovations $\varepsilon_{t+1}$ in the observables, defined in (24), relate to the underlying structural innovations, $u_{t+1}$ by

$$
\left.\varepsilon_{t+1}\right|_{\xi_{t}=\widehat{\xi}_{t}}=H_{\xi}^{\prime}\left(\xi_{t+1}-\left.E_{t} \xi_{t+1}\right|_{\xi_{t}=\widehat{\xi}_{t}}\right)=H_{\xi}^{\prime} F_{u} u_{t+1}
$$

If the first two conditions hold, $H_{\xi}^{\prime} F_{u}$ is both square $(n=s)$ and invertible. Then if the Kalman filter reveals the true states in period $t$, the structural innovations can be derived from the innovations to the observables by inverting (25). Hence the states will also be known in period $t+1$ and so on indefinitely.

However, this simply tells us that if the first two conditions in Proposition 3 are satisfied, then $M=0$, (and hence, from (19), $P=Q$ ) is a fixed point of the Kalman filter. The third condition tells us whether this fixed point is stable and shows that the nature of the measurement process and its interaction with the dynamics of the underlying states, is crucial. The intuition for this third condition is discussed below, after Corollary $2 .^{18}$

The conditions set by Proposition 3 state the minimal conditions necessary for the assumption of full information to be at least asymptotically valid. All three conditions can be related to interesting economic features of DSGE models.

1. Asymptotic invertibility is only of interest in the case that $s$, the stochastic dimension of an economic system, is lower than $r$, the dimension of the states. The benchmark stochastic growth model, for example, is driven by a single stochastic process for technology. The first condition in Proposition 3 implies that in this economy (subject to the other two conditions also holding) full information can be replicated, at least asymptotically, with only a single observable variable. We illustrate this property in Section 6.

2. A long-recognized inference problem (Muth, 1961, Bomfim, 2004), arises when technology is subject to shocks with different persistence. Since such shocks

\footnotetext{
${ }^{17}$ This assumption is made purely to simplify the algebra; the same qualititative properties hold with both forms of endogeneity.

${ }^{18}$ In Appendix E we show that the three conditions in Proposition 3 have a mathematical counterpart to an invertibility condition for an econometrician stated in Fernandez-Villaverde et al. (2007).
} 
have an identical initial impact on technology, they will also typically affect all observable variables identically on impact. In such cases, even if the first condition in Proposition 3 is satisfied, so that there are as many observable variables as shocks, the second condition will not be satisfied (i.e. if $H_{\xi}^{\prime} F_{u}$ is not invertible). Thus inference problems of this type will be endemic to a wide range of DSGE models.

3. Even if both the first two conditions are satisfied, the third condition, relating to the nature of the measurement process, can also be crucial. In Section 6 we give an example, again in the context of the stochastic growth model, in which the information set satisfies conditions 1 and 2, but does not satisfy condition 3 and therefore is not asymptotically invertible.

While a system with an asymptotically invertible information set will converge to a solution in which the states are perfectly observable, for finite $t$ it will display differences from the full information solution. We discuss these issues further in Sections 4.2 and 4.4 .

\section{Non-invertible information sets}

We now address the implications of an information set being non-invertible. We first show that even in this case the observable dynamics of the economy can be represented by those of a notional full information economy, but with a different covariance pattern of innovations. We then examine the implied true (but, in real time, intrinsically unobservable) dynamics of the economy, which are more complex.

\subsection{An isomorphic representation}

Corollary 1 Assuming convergence of the Kalman filter, the behaviour of the estimated states $\widehat{\xi}_{t}$ and forward-looking variables $c_{t}$ is isomorphic to the behaviour of the true states, $\xi_{t}$ and $c_{t}$ under full information, if $Q$, defined in (11) as the covariance matrix of the innovations $v_{t}$ in (7), is replaced by the matrix $Q+F_{\xi} M F_{\xi}^{\prime}-M$. In this representation there will in general be "pseudo-shocks" to states that are in reality pre-determined.

Proof. See Appendix C.

This property follows directly from the representation of the state estimates in (22), which shows that the $\widehat{\xi}_{t}$ have an autoregressive representation which is identical that of the full information states, $\xi_{t}^{*}$ in (13) except in terms of its innovations. If 
the Kalman filter has converged to its steady state, the innovations to the estimated states are a linear combination of the innovations to the observables and are vector white noise conditional upon the $t$ - dated information set with a time-invariant distribution. Furthermore, given the certainty equivalent nature of the forwardlooking variables $c_{t}$ in (16), the estimated states $\widehat{\xi}_{t}$ are sufficient for a time series representation of $c_{t}$ under any information set, just as the true states are under full information. $^{19}$

However, despite its identical autoregressive representation, the notional full information economy described by Corollary 1 will have different dynamic properties, due to the nature of its innovations. Most notably, as stated in Corollary 1, there will in general be "pseudo-shocks" to predetermined variables that are logically impossible under full information. Thus analysis of an economy with a non-invertible information set may require the derivation of impulse responses to, for example, "capital shocks" that are never considered in the standard analysis of models with full information. ${ }^{20}$

Given certainty equivalence, any differences in the dynamics of the states in the isomorphic representation in Corollary 1 in turn determine the impact of noninvertible information sets on the time series properties of forward-looking variables. The additional terms in the innovation covariance matrix of the isomorphic representation do not always sum to a positive definite matrix. Hence non-invertibility of an information set can in principle result in a process for forward-looking variables with higher, or lower, variance than under full information. ${ }^{21}$

\subsection{True state dynamics and the implications of non-invertibility}

While there is, as stated in Corollary 1, an autoregressive representation of the state estimates in terms of observable innovations, this representation does not describe the dynamics of the true states, except in the special case of invertibility. For the general non-invertible case the true (but unobservable) dynamics of the economy are richer once expressed in terms of the true structural innovations, $u_{t}$. Analysis of these dynamics provides further important insights.

\footnotetext{
${ }^{19}$ This reduced form representation of the estimated states has an econometric parallel in the "Innovations Representation" of Hansen and Sargent (2005, p 191).

${ }^{20}$ In reality these are of course not true shocks, but an amalgam of true shocks and revisions to past estimates of the states: but Corollary 1 implies that the responses of the forward-looking variables, and hence of the states, to these pseudo-shocks will be identical to the full information response, if such shocks were actually possible.

${ }^{21}$ A point also made by Pearlman et al. (1986) and Pearlman (1992) and relevant to the results of Bomfim (2004).
} 
Define the vector of state filtering errors as

$$
f_{t} \equiv \xi_{t}-\widehat{\xi}_{t}
$$

We show in Appendix $\mathrm{D}$ that the joint process for $f_{t}$ and the true states $\xi_{t}$ can be expressed, for any information set, in the time-varying vector autoregressive form

$$
\left[\begin{array}{c}
\xi_{t+1} \\
f_{t+1}
\end{array}\right]=\left[\begin{array}{cc}
G & -F_{c} \eta^{\prime} \\
0 & \left(I-\widetilde{\beta}_{t} H_{\xi}^{\prime}\right) F_{\xi}
\end{array}\right]\left[\begin{array}{c}
\xi_{t} \\
f_{t}
\end{array}\right]+\left[\begin{array}{c}
I \\
I-\widetilde{\beta}_{t} H_{\xi}^{\prime}
\end{array}\right] F_{u} u_{t+1} \in \mathbb{R}^{2 r \times 1}
$$

The top block is entirely independent of filtering parameters and transparently reduces to the full information process (13) when filtering error $f_{t}$ is zero. In general, with dynamic endogeneity $\left(F_{c} \neq 0\right)$, filtering error "contaminates" state dynamics via the off-diagonal element of the autoregressive matrix for the joint process for $\xi_{t}$ and $f_{t}$. In contrast the process for the vector of state filtering errors $f_{t}$ is block recursive. Furthermore, consistent with Proposition $1, f_{t}$ follows an identical process to the state filtering error in the parallel exogenous problem (i.e. it does not depend on $F_{c}$ or $H_{c}$ ) and hence is also invariant to the properties of the $c_{t}$, the forward-looking variables. Note that while the autoregressive matrix of $f_{t}$ is time-varying away from steady state, due to time variation in $\beta_{t}$, this time variation is deterministic.

Proposition 2 has an important corollary that is crucial to the time series properties summarised in (27):

Corollary 2 Assuming convergence of the Kalman filter, in the autoregressive representation (27), the steady-state autoregressive matrix $\left(I-\widetilde{\beta} H_{\xi}^{\prime}\right) F_{\xi}$ of the filtering errors, $f_{t} \equiv \xi_{t}-\widehat{\xi}_{t}$, has at most $r-n$ non-zero eigenvalues, all of which have real parts strictly less than unity in absolute value.

Proof. See Appendix C.

Thus convergence of the Kalman filter to a unique steady state automatically implies that filtering errors are stationary in the neighbourhood of the steady state and vice versa.

Corollary 2 sheds light on the third condition for asymptotic invertibility in Proposition 3. If the first two conditions are satisfied there is a fixed point of the Kalman filter that replicates full information. But the third condition in Proposition 3 can be interpreted as a requirement that the full information fixed point be stable, since from Corollary 2 this is equivalent to a condition that the eigenvalues of the autoregressive matrix of filtering errors in (27) be stable in the neighbourhood of the solution that replicates full information. Furthermore, since, using the 
definitions of $f_{t}$ and $M_{t}$ in (26) and (17), we have $M_{t}=E\left(f_{t} f_{t}^{\prime}\right)$, from Definition 3, asymptotic invertibility implies $\lim _{t \rightarrow \infty} M_{t}=M=0$, so that if all three conditions in Proposition 3 are met the filtering errors $f_{t}$ have a degenerate distribution.

For the general non-invertible case, in which the conditions in Proposition 3 are not met, $f_{t} \neq 0$ and hence filtering error will always "contaminate" state dynamics. Assuming the conditions in Proposition 2 hold, however, there will still be a steady state of the Kalman filter, in the neighbourhood of which, from Corollary 2, filtering errors will be stationary. But this steady state will not replicate full information.

The joint process for $\xi_{t}$ and $f_{t}$ in (27) provides a complete description of the true process for the forward-looking variables $c_{t}$, since, using (16) we can write $c_{t}=\eta^{\prime} \widehat{\xi}_{t} \equiv \eta^{\prime}\left(\xi_{t}-f_{t}\right)$ : thus with a non-invertible information set the process for $c_{t}$ differs from the process under full information both because of the direct effect of filtering error on the estimated states and because the true states differ from their full information values. ${ }^{22}$ The more persistent is the filtering error process (the closer are the non-zero eigenvalues of $\left(I-\widetilde{\beta} H_{\xi}^{\prime}\right) F_{\xi}$ to unity), the more prolonged will be the additional dynamics introduced by the filtering problem.

Note that, while filtering errors will contaminate the true state dynamics, they will not do so in expectation, since $E_{t} f_{t}=0$ by definition. Given this, at any point in time the expected process for the states has an identical form to that of the true states under full information, hence the undetermined coefficients problem to which $\eta$ is the solution is identical to that under full information. This feature verifies the assumption of certainty equivalence used in the solution for the forward-looking variables, $c_{t}$, in (16). ${ }^{23}$

The representation in (27), together with Corollary 2, also implies a number of further features of non-invertible information sets that are direct corollaries of Propositions 1 and 2, given Assumptions 1 and 2.

Corollary 3 Non-invertibility of an information set has no permanent effects, even when there are permanent structural shocks (i.e. if $B_{z z}$ has one or more unit eigenvalues).

Proof. See Appendix D

\footnotetext{
${ }^{22}$ Note that only filtering errors in the underlying states $k_{t}$ and $z_{t}$ have any direct impact on $c_{t}$ since, as noted previously, $\eta$ has zeros in its $i$ th row for $i>r_{k}+r_{z}$.

${ }^{23}$ In a DSGE model, certainty equivalence is of course a standard result that arises from the fact that we first linearise the model (including Euler equations) and then solve the filtering problem. To the extent that state uncertainty introduces new sources of variance in forward-looking variables (an issue we discuss in Section 4.3) incorporation of state uncertainty into the optimisation problem before linearisation would presumably result in effects analogous to those in the precautionary saving literature.
} 
Corollary 3 implies that impulse responses under full information and noninvertible information must converge. Thus while short-run dynamics may be significantly affected by informational problems, longer-run responses (especially those to permanent shocks) derived from models that assume full information will approximate those from models with non-invertible information sets. This has potentially important implications for econometric analysis: it means, for example, that identifying assumptions for vector autoregressive models based on long-run restrictions predicated on theoretical models assuming full information are equally applicable under imperfect information.

Corollary 3 also implies that in the case of asymptotic invertibility as in Proposition 3, the impact of any filtering errors away from steady state must ultimately decay to zero, so that in this case $\xi_{t} \rightarrow \xi_{t}^{*}:$ in the limit the full information solution is replicated.

Corollary 4 The filtering errors $f_{t}$ satisfy $H_{\xi}^{\prime} f_{t}=0$.

\section{Proof. See Appendix D}

Linear dependence between the elements of the vector of filtering errors arises from the efficient use of the structural knowledge of the economy that underpins the Kalman filter. To see the intuition for this result, note that, if we take $t$ - dated expectations of the measurement equation (8), using (12) this implies

$$
\begin{aligned}
i_{t} & =H_{\xi} \xi_{t}+H_{c} \eta^{\widehat{\xi}_{t}}=\left(H_{\xi}+H_{c} \eta^{\prime}\right) \widehat{\xi}_{t} \\
& \Rightarrow H_{\xi}^{\prime} \xi_{t}=H_{\xi}^{\prime} \widehat{\xi}_{t} \Rightarrow H_{\xi} f_{t}=0
\end{aligned}
$$

thus filtering errors for any given state variable must be precisely offset by some combination of other filtering errors. By implication neither the innovation matrix of the vector of filtering errors, $f_{t}$, nor its autoregressive matrix can be of full rank and thus the $n$ - dimensional vector $f_{t}$ can always be expressed in terms of a subvector of dimension $r-n$.

Corollary 5 Let $\beta=\left[\begin{array}{lll}\beta_{k} & \beta_{z} & \beta_{w}\end{array}\right]^{\prime}$. If $F_{\xi}$ has explosive eigenvalues, $\beta_{k}=0$ can never be a convergent solution of the Kalman filter problem.

Proof. See Appendix D

This result follows directly from the stationarity of the filtering error process. When $F_{\xi}$ has explosive eigenvalues associated with the evolution of the pre-determined variables, $k_{t}$, the steady-state autoregressive matrix of the filtering error in (27), $\left(I-\widetilde{\beta} H_{\xi}^{\prime}\right) F_{\xi}$, could not have stable eigenvalues with $\widetilde{\beta}_{k}=0$. Since, from Corollary 
2 , stability of the filtering errors is directly equivalent to convergence of the Kalman filter, this in turn implies that in this case $\beta_{k} \neq 0$ can never be a solution of the filtering problem.

This has interesting implications for the nature of the optimal response to information, as the quality of that information deteriorates. In standard exogenous Kalman filter problems, in which $F_{c}=0$ and $F_{\xi}$ usually has at worst borderline unit eigenvalues, the lower the quality of the information, the smaller is the optimal response to that information. The same feature applies in our problem when $F_{\xi}$ has stable or unit eigenvalues. ${ }^{24}$ In contrast, if $F_{\xi}$ has explosive eigenvalues, so does the state process in the parallel problem of Proposition 1 . So for $\beta_{k}$ sufficiently close to zero, the filtering error process would itself be explosive, contradicting Corollary 2. In this case, as $S_{\omega \omega}$ tends to infinity, $\beta_{k}$ tends to a fixed, non-zero matrix. Thus however poor the information, it is always optimal to respond to it. ${ }^{25}$

\subsection{A caveat on impulse responses}

The joint process for $\xi_{t}$ and $f_{t}$ in (27) discussed in the previous section shows that non-invertible information introduces more complicated dynamics than under full information. However, impulse responses derived from the full reduced form representation in (27) would not be observable in real time. The only observable impulse responses would be those to the isomorphic representation of Corollary 1, in which, as already discussed, the innovation covariance properties of this notional economy may be very different from those of the true structural shocks. ${ }^{26}$

\subsection{Mappings}

We have discussed four types of information set: full information; instantaneous invertibility; asymptotic invertibility and general incomplete information. These can be contrasted by writing the system as mappings, either from initial values and shocks to the true states, or from observables to state estimates.

\footnotetext{
${ }^{24}$ This feature is noted in Svensson and Woodford (2003, p711) where it appears to be presented as a general result.

${ }^{25}$ A close parallel is in Sims (2003, p680) who notes that under rational inattention, if the response of consumption is too weak to stabilise the explosive states there will be no stationary equilibrium.

${ }^{26}$ There is a counterpart here with the econometric invertibility problem in Fernandez-Villaverde et al. (2007).
} 


\subsubsection{The mapping from initial values and shocks to the true states}

Inputs consisting of initial values $\xi_{0}, \Phi_{0}=\left\{\widehat{\xi}_{0}, P_{0}\right\}$ and shocks $\left\{u_{i}\right\}_{i=1}^{t}$ are mapped to outputs consisting of states $\left\{\xi_{i}\right\}_{i=1}^{t}$ and filtering errors $\left\{f_{i}\right\}_{i=1}^{t}$, by (19) - (21) and (27), reproduced here

$$
\begin{aligned}
& \widetilde{\beta}_{t}=P_{t} H_{\xi}\left(H_{\xi}^{\prime} P_{t} H_{\xi}\right)^{-1} \in \mathbb{R}^{r \times n} \\
& M_{t}=\left(I_{r}-\widetilde{\beta}_{t} H_{\xi}^{\prime}\right) P_{t} \in \mathbb{R}^{r \times x} \\
& P_{t+1}=F_{\xi} M_{t} F_{\xi}^{\prime}+Q \in \mathbb{R}^{r \times r} \text { where } Q=F_{u} S F_{u}^{\prime} \\
& {\left[\begin{array}{c}
\xi_{t+1} \\
f_{t+1}
\end{array}\right]=\left[\begin{array}{cc}
G & -F_{c} \eta^{\prime} \\
0 & \left(I-\widetilde{\beta}_{t} H_{\xi}^{\prime}\right) F_{\xi}
\end{array}\right]\left[\begin{array}{c}
\xi_{t} \\
f_{t}
\end{array}\right]+\left[\begin{array}{c}
I \\
I-\widetilde{\beta}_{t} H_{\xi}^{\prime}
\end{array}\right] F_{u} u_{t+1} \in \mathbb{R}^{2 r \times 1}}
\end{aligned}
$$

The four types of information set are:

1. Full information: under full information $\Phi_{0}=\left\{\xi_{0}, Q\right\}, P_{t}=Q, M_{t}=$ $0, f_{t}=0, \forall t \geq 0$ and the mapping reduces to

$$
\xi_{t+1}=G \xi_{t}+F_{u} u_{t+1}
$$

which, given (10) is identical to the standard solution (13).

2. Instantaneous invertibility: using (15) and (16) we have

$$
\widehat{\xi}_{t}=\left(I+\left(H_{\xi}^{\prime}\right)^{-1} H_{c} \eta^{\prime}\right)^{-1}\left(H_{\xi}^{\prime}\right)^{-1} i_{t}=\xi_{t} \quad \forall t \geq 0
$$

Hence observing $i_{t}$ is equivalent to observing $\xi_{t}$ and again, $\Phi_{0}=\left\{\xi_{0}, P_{0}\right\}, M_{0}=$ 0 , so this case is identical to full information.

3. General incomplete information: $\Phi_{0}=\left\{\widehat{\xi}_{0}, P_{0}\right\}$. Under the conditions stated in Proposition 2 initial estimates have only transitory impact and the Kalman Filter converges to a unique steady state. Thus as $t \rightarrow \infty, P_{t} \rightarrow P$, $M_{t} \rightarrow M, \widetilde{\beta}_{t} \rightarrow \widetilde{\beta}(P)$ and the mapping converges to a time invariant vector autoregressive process.

4. Asymptotic invertibility: as for case 3 , but with $\lim _{t \rightarrow \infty} P_{t}=P=Q, \lim _{t \rightarrow \infty} M_{t}=$ $M=0, \lim _{t \rightarrow \infty} \widetilde{\beta}_{t}=\widetilde{\beta}(Q)=F_{u}\left(H_{\xi}^{\prime} F_{u}\right)^{-1}$ thus $\lim _{t \rightarrow \infty} f_{t}=0$, so that in the limit as $t \rightarrow \infty$ the mapping reduces to (33). For finite $t$, the behaviour of the model will in general be different from the full-information case, since $P_{t}-Q$ is non-negative definite symmetric. 


\subsubsection{The mapping from initial values and observables to state estimates}

Whereas the above mapping was in terms of quantities that will in general be unobservable, there is also a mapping in terms of observables. Inputs, consisting of initial values $\widehat{\xi}_{0}$ and $P_{0}$ and observables $\left\{i_{i}\right\}_{i=0}^{t}$, are mapped to outputs, consisting of state estimates $\left\{\widehat{\xi}_{i}\right\}_{i=1}^{t}$, by

$$
\begin{gathered}
E_{t} i_{t+1}=\left[H_{\xi}^{\prime}+H_{c} \eta^{\prime}\right] G \widehat{\xi}_{t} \in \mathbb{R}^{n \times 1} \\
\widehat{\xi}_{t+1}=G \widehat{\xi}_{t}+\beta_{t}\left[i_{t+1}-E_{t} i_{t+1}\right] \in \mathbb{R}^{r \times 1}
\end{gathered}
$$

where $\beta_{t}$ is defined as above in (19) - (21), (24).

The four cases are:

1. Full information: we have $\widehat{\xi}_{t}=\xi_{t}, \beta_{t}=I_{r}$ and $i_{t+1}-E_{t} i_{t+1}=F_{u} u_{t+1}$, so that $(36)$ reduces to $(33)$

2. Instantaneous invertibility: since $\xi_{t}$ can be derived directly from $i_{t}$ (as in the previous mapping) the mapping is identical to that in Case 1.

3. General incomplete information: under the assumptions of Proposition 2 , as $t \rightarrow \infty \beta_{t} \rightarrow \beta$ and hence the mapping reduces to a time invariant vector autoregressive representation of the state estimates, which in turn, from Corollary 1, is isomorphic to a full information state process with "pseudo shocks".

4. Asymptotic invertibility: $\widetilde{\beta}_{t}$ converges to $\widetilde{\beta}(Q)=F_{u}\left(H_{\xi}^{\prime} F_{u}\right)^{-1}$, so that as $t \rightarrow \infty$ the structural shocks $u_{t}$ can be recovered from the innovations to the observables.

In both cases 3 and 4 when the Kalman filter has not converged $(t<\infty)$, there

will be "pseudo-shocks" to state estimates. However these are different from the pseudo-shocks in the converged case in that they will be drawn from a time-varying (and in case 4, degenerate) distribution

\section{Time series representations of the observables}

It is a standard result (see Hamilton, 1994, p. 391) that, conditional upon convergence of the Kalman filter, the innovations $\varepsilon_{t}$ to the observable variables $i_{t}$ must also be the innovations to an invertible, or fundamental, time series representation 
of $i_{t}$. The key characteristic of fundamental innovations is that they can be recovered from and are white noise conditional upon, the history of $i_{t}$. The link with the Kalman filter is evident from the fact that the same applies for the innovations that condition upon state estimates, $\widehat{\xi}_{t}$.

Another standard result in time series analysis (Hamilton, 1994, pp. 67-68; Lippi and Reichlin, 1994) is that for any fundamental autoregressive moving average representation of an observable vector process such as $i_{t}$ there is a finite set of alternative representations of the same ARMA order, but each with a different set of nonfundamental innovations. These nonfundamental representations have the same autocovariance properties as the fundamental representation, but the associated innovations are not recoverable from the history of $i_{t}$.

A further feature of time series representations noted by Lippi and Reichlin (1994) is that for any fundamental representation there is a potentially infinite set of nonfundamental representations of arbitrarily higher ARMA order, which they term non-basic. ${ }^{27}$ Lippi and Reichlin conclude however that such non-basic representations are "not likely to occur in models based on economic theory" (Lippi and Reichlin 1994, p. 315).

These characteristics of the time series representation of $i_{t}$ are invariant to the nature of the information set or of the underlying structural model. Where the nature of the information set and the structural model does make a difference is in the nature of the link between the alternative time series representations of $i_{t}$ and the underlying structural innovations, $u_{t}$ to the true state process. The following proposition summarises the nature of this link.

Proposition 4 (time series representations and structural innovations) For any $n \times 1$ vector of observables, $i_{t}$ that satisfies the measurement equation (8) and given convergence of the Kalman filter to its steady state:

a)For any information set $I_{t}$ there exists a fundamental vector autoregressive

${ }^{27}$ The intuition for non-basic representations can be demonstrated by noting that any univariate white noise process can in principle by written as a nonfundamental $\operatorname{ARMA}(1,1)$ in terms of another white noise process with lower variance. For example, if $\omega_{t}$ is univariate white noise, for any arbitrary $\psi \in(-1,1)$ we can write

$$
\omega_{t}=\left(\frac{1-\psi^{-1} L}{1-\psi L}\right) \zeta_{t}
$$

with $\sigma_{\zeta}^{2}=\psi^{2} \sigma_{\omega}^{2}$. The fundamental representation has MA parameter equal to $\psi$, and hence the MA and AR components cancel, which indeed they must, since $\omega_{t}$ is univariate white noise. Thus the the history of $\omega_{t}$ can be consistent with any value of $\psi$ and hence any value of $\sigma_{\zeta}^{2}$. Furthermore, for any arbitrary choice of $\psi \neq 0$ the nonfundamental innovation $\zeta_{t}$ can in turn be given a nonfundamental representation, and so on ad infinitum. Hence in principle there is an infinite set of nonfundamental representation of $\omega_{t}$, of arbitrary order, but they are all nonbasic. 
moving average $\operatorname{VARMA}(n, p, q)$ representation of $i_{t}$ with autoregressive order $p \leq$ $r-n+1$ and moving average order $q \leq r-n$.

b) If $I_{t}$ is invertible, the structural innovations $u_{t}$ are, up to a scaling factor, the innovations to this fundamental representation.

c) If $I_{t}$ is non-invertible and $n=s=\operatorname{rank}\left(H_{\xi}^{\prime} F_{u}\right)$ the $u_{t}$ are, up to a scaling factor, innovations to a non-fundamental representation of $i_{t}$

d) If $I_{t}$ is non-invertible and $n<s$, the innovations to nonfundamental representations can in general only be recovered from an information set consisting of both the history and future of the structural innovations, $u_{t}$.

e) If $I_{t}$ is non-invertible and there is dynamic endogeneity $\left(F_{c} \neq 0\right)$, the true process for $i_{t}$ implies a nonbasic nonfundamental VARMA representation with reciprocal autoregressive roots given by the eigenvalues of $G$ and the eigenvalues of $\left(I-\widetilde{\beta} H_{\xi}^{\prime}\right) F_{\xi}$.

Proof. See Appendix F

Table 1 summaries the properties of the information set and the resulting time series representation of $i_{t}$.

Part (a) of the proposition states that the fundamental time series representation of the observables has autoregressive and moving average orders reducing in $n$, the dimension of the observables. In the special case of instantaneous invertibility $(r=n)$ Table 1 shows that both the states and the observables have a first order VAR representation, the reciprocal autoregressive roots of which are the eigenvalues of $G$, defined in (14).

Part (b) covers the cases of both instantaneous and asymptotic invertibility. In the first case, described in the first row of Table 1, we can recover the true states, $\xi_{t}$ from (15), substitute for $c_{t}$ and re-write the law of motion for $\xi_{t}(7)$ as a vector autoregression for $i_{t}$. In the second case of asymptotic invertibility (as in Proposition 3 ), the $u_{t}$ can be recovered from the $\varepsilon_{t}$, but, from part (a) of the Proposition, a pure VAR representation of $i_{t}$ in terms of $u_{t}$ does not exist, since $s<r$. It is however possible to derive a finite order VARMA representation by substituting for $\varepsilon_{t}$ in terms of $u_{t}$ using (25), see the second row of Table 1 . In both cases, since $u_{t}$ can be recovered from the history of the observables, $i_{t}$, invertibility of the information set and fundamentalness of the $u_{t}$ are two sides of the same coin.

If the information set is non-invertible, the vector of structural innovations $u_{t}$ cannot be recovered from the history of the observables. Part (c) of the proposition highlights an interesting special case, when $s$, the number of structural innovations equals $n$, the number of linearly independent observables, but the information set is not asymptotically invertible (i.e. the third condition in Proposition 3 is not 
satisfied). In this case a VARMA representation of $i_{t}$ with innovations given by some scaling of $u_{t}$ does exist, but these innovations must be nonfundamental. The nonfundamentalness of $u_{t}$ in this case is just another way of saying that, despite the fact that both sets of innovations have the same dimension, the $u_{t}$ cannot be recovered from the history of the observables.

In the more general non-invertible case, in which $n<s$, summarised in part (d) of the proposition, the link between VARMA and structural innovations is less straightforward but is included for the sake of completeness. ${ }^{28}$

Part (e) reveals an aspect of the time series properties of the observables that is specific to the endogenous Kalman Filter. As discussed in Section 4, the true state process in (27) contains additional dynamics due to the contamination of the state process by filtering error due to dynamic endogeneity (when $F_{c} \neq 0$ ). These additional dynamics are not observable: so the fundamental reduced form VARMA representation is of the same order as the reduced form of the system under full information (since it can be written in terms of the isomorphic state representation of Corollary 1). However, there is a nonfundamental VARMA representation that contains these additional dynamics: as a result this representation is not only nonfundamental, but also nonbasic in Lippi and Reichlin's (1994) terminology. Thus, in contrast, to Lippi and Reichlin's conclusion that nonbasic nonfundamental representations are unlikely to occur in models based on economic theory, part (e) shows that, on the contrary, they are an intrinsic feature of models that combine endogenous states with non-invertible information sets. When the states are exogenous, however (if $F_{c}=0$ ), this feature disappears. The last two rows of Table 1 illustrate the contrast between the cases of exogenous and endogenous states.

The nonbasic nature of these representations is not just of theoretical interest. If nonfundamental representations are at least basic, this implies that, while the structural innovations themselves cannot be derived from the history of the observables, it is at least possible for an econometrician to identify from the data some of the characteristics (e.g. covariance properties and impulse responses) of these unob-

\footnotetext{
${ }^{28}$ The contrast between the cases in (c) and (d) can be be briefly summarised as follows. When $n=s$, as in part (c) of the proposition, we have $\varepsilon_{t}=A(L) u_{t}$, where $A(L)$ is a square matrix lag polynomial that is a reduced form of the true state process in (27). While $A(L)$ is non-invertible in non-negative powers of $L$ it is invertible in the forward operator hence to recover $u_{t}$ requires information on the future, as well as the history of $\varepsilon_{t}$, and hence of the observables. When $n<s$, as in part (d) of the proposition, $A(L)$ is no longer square. But any nonfundamental representation also has a set of innovations $\eta_{t}$ of the same dimension as $\varepsilon_{t}$ such that $\varepsilon_{t}=B(L) \eta_{t}$, where $B(L)$ is square. Thus $\eta_{t}=B(L)^{-1} A(L) u_{t}$, where the inversion of $B(L)$ again involves a term in the forward operator and hence $\eta_{t}$ depends in general on both the history and the future of the structural innovations. Only when $n=s$, as in part (c), do we have $B(L)=A(L)$, so that these additional dynamics cancel out.
} 
servable structural innovations from the observable fundamental representation. The non-basic characteristic of VARMA representations that arise from non-invertible information sets with endogenous states rules this out.

\section{Example: information in the stochastic growth model}

In this section we present an analytical example that shows how our techniques can be applied to the benchmark stochastic growth model. We consider three aggregate information sets corresponding to the three cases of instantaneous invertibility, asymptotic invertibility and non-invertibility.

Following Campbell (1994) the aggregate state evolution equations are

$$
\begin{aligned}
& k_{t+1}=\lambda_{1} k_{2}+\lambda_{2} a_{t}+\left(1-\lambda_{1}-\lambda_{2}\right) c_{t} \\
& a_{t+1}=\phi a_{t}+u_{t+1}
\end{aligned}
$$

where capital $k_{t}$ is pre-determined, $a_{t}$ is aggregate technology and $c_{t}$ is consumption and the $\lambda_{i}$ are linearisation constants.

Implicitly underlying the aggregates is a heterogeneous agent model in which each agent faces a budget constraint of the form (37) but in idiosyncratic variables. We do not need to give details of this model (although we discuss it further in Section 6.4) since we are only concerned with the circumstances under which information on aggregate prices, assumed to be common knowledge, is or is not sufficient to reveal aggregate states.

Note that the aggregate budget constraint will hold at all times and in all states of nature irrespective of how individual consumption is determined and whatever the level of aggregate consumption. Even if this means linearised capital falls without bound, aggregate capital in levels is bounded below by zero.

If we define the state vector as $\xi_{t}=\left[\begin{array}{ll}k_{t} & a_{t}\end{array}\right]^{\prime}$, we can rewrite (37) and (38), using (10), in the form of (7) as:

$$
\xi_{t+1}=F_{\xi} \xi_{t}+F_{c} c_{t}+F_{u} u_{t+1}
$$

where

$$
F_{\xi}=\left[\begin{array}{cc}
\lambda_{1} & \lambda_{2} \\
0 & \phi
\end{array}\right] ; F_{c}=\left[\begin{array}{c}
1-\lambda_{1}-\lambda_{2} \\
0
\end{array}\right] ; F_{u}=\left[\begin{array}{l}
0 \\
1
\end{array}\right]
$$

Using Campbell's (1994, p. 469) derivation of the linearisation constants from un- 
derlying structural parameters, ${ }^{29}$ we have $\lambda_{2}=\alpha(r+\delta) /((1+g)(1-\alpha))>0$, where $r$ is the steady state value of the return on capital, $g$ is the growth rate, $\delta$ is the depreciation rate and $\alpha$ is the exponent on labour in a Cobb-Douglas production function. The eigenvalues of $F_{\xi}$ are $\phi \in[0,1]$ and $\lambda_{1}=\frac{1+r}{1+g}$. Under the standard assumptions of dynamic efficiency, therefore, $r>g \Rightarrow \lambda_{1}>1$ hence $F_{\xi}$, the autoregressive matrix of the states in the parallel problem of Proposition 1, has one explosive eigenvalue.

We now consider what information is available to agents in the model. The standard assumption that $k_{t}$ and $a_{t}$ can both be directly measured is arguably a questionable one. ${ }^{30}$ It appears more reasonable to assume that the information set is "market-consistent" (Graham and Wright, 2010): that agents have information based on the prices in the markets they trade in. Using this assumption, the vector of possible observable aggregate variables is:

$$
\left[\begin{array}{c}
w_{t} \\
r_{t}
\end{array}\right]=\left[\begin{array}{cc}
1-\alpha & \alpha \\
-\lambda_{3} & \lambda_{3}
\end{array}\right]\left[\begin{array}{c}
k_{t} \\
a_{t}
\end{array}\right]
$$

where $w_{t}$ is the aggregate wage, $r_{t}$ is the aggregate return on capital and $\lambda_{3}>0$ is Campbell's third linearisation parameter. The first line of (41) is the marginal product relation for labour, the second uses Campbell's linearisation of the return on capital. There is no noise in the measurement process.

We now consider the implications of three possible information sets. The first assumes that both possible measured variables are observable. The other two are censored versions of the information set, in which there is only a single observable variable.

The apparently arbitrary nature of these censored information sets can be justified on several grounds. First, they provide a technical illustration that contrasts two simple cases in which asymptotic invertibility does and does not hold, which we show relates to the nature of the measurement process and its interaction with the evolution of the states. Second, they are of some independent interest, since in any solution that does asymptotically replicate full information the pre-determined nature of capital in this model means that innovations to the two measured variables will be perfectly correlated. The examples therefore allow us to identify which of

\footnotetext{
${ }^{29}$ We simplify the specification of the law of motion of capital and the measurement process by following Campbell's assumption in the first half of his paper that labour supply is fixed. This means that we have only one of the two potential forms of endogeneity in the filtering problem $\left(H_{c}=0\right.$ but $\left.F_{c} \neq 0\right)$. Given that we can exploit the "parallel problem" of Proposition 1 in solving the filtering problem, setting $H_{c}=0$ has no qualitative impact on the results.

${ }^{30}$ For a discussion of this assumption see Bomfim (2004), Porapakarrm and Young (2008) and Graham and Wright (2010).
} 
the two observable variables will be informationally redundant if full information can be replicated. Third, as we discuss in Section 6.4, each restricted information set can be derived as a special case of fully specified models.

\subsection{An instantaneously invertible information set}

In the first case we assume that $i_{t}=\left[\begin{array}{ll}w_{t} & r_{t}\end{array}\right]^{\prime}$. Given this information set, we have $n=r=2$ and by inspection of (41) the conditions for instantaneous invertibility in Definition 2 are satisfied. Thus factor prices provide sufficient information to infer the state vector from $t$-dated information and hence the Kalman filter is redundant.

\subsection{An asymptotically invertible information set}

We first consider $i_{t}=w_{t}$, hence $H_{\xi}^{\prime}=\left[\begin{array}{ll}1-\alpha & \alpha\end{array}\right]$. With a single shock, we thus have $n=s=1$, hence the first condition in Proposition 3 is satisfied. The second is also satisfied, since $\left(H_{\xi}^{\prime} F_{u}\right)^{-1}=\left(\left[\begin{array}{ll}1-\alpha & \alpha\end{array}\right]\left[\begin{array}{ll}0 & 1\end{array}\right]^{\prime}\right)^{-1}=\alpha^{-1} \neq 0$. For the third condition, we have, using the formulae in the proposition,

$$
\begin{aligned}
\widetilde{\beta}(Q) & =F_{u}\left(H_{\xi}^{\prime} F_{u}\right)^{-1}=\left[\begin{array}{l}
0 \\
1
\end{array}\right] \frac{1}{\alpha}=\left[\begin{array}{c}
0 \\
\frac{1}{\alpha}
\end{array}\right] \\
\left(I-\widetilde{\beta}(Q) H_{\xi}^{\prime}\right) F_{\xi} & =\left(\left[\begin{array}{cc}
1 & 0 \\
0 & 1
\end{array}\right]-\left[\begin{array}{c}
0 \\
\frac{1}{\alpha}
\end{array}\right]\left[\begin{array}{cc}
1-\alpha & \alpha
\end{array}\right]\right)\left[\begin{array}{cc}
\lambda_{1} & \lambda_{2} \\
0 & \phi
\end{array}\right] \\
\Lambda\left(\left(I-\widetilde{\beta}(Q) H_{\xi}^{\prime}\right) F_{\xi}\right) & =\lambda_{1}-\left(\frac{1-\alpha}{\alpha}\right) \lambda_{2}
\end{aligned}
$$

and thus, again, using Campbell (1994) the single critical eigenvalue ${ }^{31}$ is, in terms of structural parameters,

$$
\Lambda=\lambda_{1}-\left(\frac{1-\alpha}{\alpha}\right) \lambda_{2}=\frac{1-\delta}{1+g}<1
$$

Hence the fixed point is stable, or, equivalently, using Corollary 2, filtering errors converge to zero in the neighbourhood of the full information equilibrium. Since it can also be shown that the model satisfies the conditions in Proposition 2, it follows that this is the unique steady state of the Kalman filter.

Thus the information set is asymptotically invertible: full information can be replicated from a sufficiently long history of the aggregate wage alone and hence the history of returns is informationally redundant.

\footnotetext{
${ }^{31}$ Recall that, from Corollary 2, the autoregressive matrix of filtering errors will have only $r-n=$ 1 non-zero eigenvalue.
} 


\subsection{A non-invertible information set}

We now consider the alternative censored information set, $i_{t}=r_{t}$, hence $H_{\xi}^{\prime}=$ $\left[\begin{array}{ll}-\lambda_{3} & \lambda_{3}\end{array}\right]$. The first two conditions in Proposition 3 are again trivially satisfied, with $n=s=1$; and $\left(H_{\xi}^{\prime} F_{u}\right)^{-1}=\left|\left[\begin{array}{ll}-\lambda_{3} & \lambda_{3}\end{array}\right]\left[\begin{array}{ll}0 & 1\end{array}\right]^{\prime}\right|=\lambda_{3}^{-1} \neq 0$. Thus full information is again a fixed point of the Kalman filter given this information set. However the third condition is not satisfied: it is not a stable fixed point. To see this, we again use the formulae in the proposition to give

$$
\begin{aligned}
\widetilde{\beta}(Q) & =F_{u}\left(H_{\xi}^{\prime} F_{u}\right)^{-1}=\left[\begin{array}{c}
0 \\
1
\end{array}\right] \frac{1}{\lambda_{3}}=\left[\begin{array}{c}
0 \\
\frac{1}{\lambda_{3}}
\end{array}\right] \\
\left(I-\widetilde{\beta}(Q) H_{\xi}^{\prime}\right) F_{\xi} & =\left(\left[\begin{array}{cc}
1 & 0 \\
0 & 1
\end{array}\right]-\left[\begin{array}{c}
0 \\
\frac{1}{\lambda_{3}}
\end{array}\right]\left[\begin{array}{ll}
-\lambda_{3} & \lambda_{3}
\end{array}\right]\right)\left[\begin{array}{cc}
\lambda_{1} & \lambda_{2} \\
0 & \phi
\end{array}\right] \\
\Lambda\left(\left(I-\widetilde{\beta}(Q) H_{\xi}^{\prime}\right) F_{\xi}\right) & =\Lambda\left(\left[\begin{array}{cc}
\lambda_{1} & \lambda_{2} \\
\lambda_{1} & \lambda_{2}
\end{array}\right]\right)=\lambda_{1}+\lambda_{2}>1
\end{aligned}
$$

Hence this restricted information set is non-invertible.

To see the intuition for this result and for the contrast with the previous example, recall that, from the representation in (27), the matrices in (42) and (43) are both the autoregressive matrices of the filtering error $f_{t}$ in the neighbourhood of an equilibrium that replicates full information. In the information set with only the history of wages filtering errors are stationary, hence any initial error in estimating the state decays back to zero. When the information set consists only of the history of the return on capital, filtering errors are explosive in the neighbourhood of full information. By implication, the history of the aggregate wage, however poorly measured, is never informationally redundant.

For this example it can again be shown that the information set does satisfy the two conditions in Proposition 2 for the existence of a unique steady state Kalman filter; but the non-invertibility of the information set rules out the possibility that this can replicate full information.

The solution to the filtering problem in this third example implies that, using Proposition $4 \mathrm{a}$ the single observable variable $r_{t}$ has a fundamental $\operatorname{ARMA}(2,1)$ representation. The order of this representation and its autoregressive roots, are identical to those under full information (and hence to the two preceding invertible cases). The moving average roots will however differ, as will the innovation $\varepsilon_{t}$. In the invertible cases this will simply be a scaling of the productivity shock, $u_{t}$; whereas in this case it will be a composite of current and lagged values of $u_{t}$. However, since $n=$ 
$s$, Proposition 4c implies that there will be an alternative nonbasic nonfundamental $\operatorname{ARMA}(3,2)$ representation of $r_{t}$, the innovation to which will simply be a scaling of $u_{t}{ }^{32}$

It is possible to show that the qualitative nature of the equilibrium for the aggregate economy that arises from this non-invertible information set is very close to that of the more complex model analyzed in Graham and Wright (2010), which we discuss further in the next section. The non-invertible information set results in "pseudo-shocks" to capital, of opposite sign to the true technology shock; this in turn induces significant differences in aggregate dynamics.

\subsection{Discussion}

Although we have discussed the three cases purely in terms of the aggregate economy, each of the three examples can be related to an underlying economy with many agents. Note that, given symmetry of information sets on the aggregate economy, it is the nature of the parallel problem that allows us to focus on aggregates, since the solution to the filtering problem is independent of exactly how any particular agent determines their consumption.

The first example can be derived from an economy with many identical agents earning the same wage and the same return. The example shows that even if these agents cannot measure the aggregate states directly, the information in the market prices they observe is sufficient to recover the states. Graham and Wright (2010) show that the same information set, defined in terms of the average wage and the return on capital, will also be common knowledge in a heterogeneous economy with complete markets.

The second example shows that, given a sufficiently long history of the economy, market-consistent information sets may reveal more information than is actually required to replicate full information, since in the limit the history of the return on capital becomes informationally redundant. This example can be seen as the limiting case of a model with noise in measuring aggregate returns on capital, due for example, to market frictions or noise traders in financial markets and shows that such noise will have no impact on the equilibrium of the stochastic growth model, as long as the history of the aggregate wage is observable. The assumption of frictionless and complete financial markets is thus not of itself a necessary condition for replication of full information.

\footnotetext{
${ }^{32}$ The AR roots in the fundamental representation will be the eigenvalues of $G$, hence will depend on the nature of the consumption function (which corresponds to (16) in the general model). The additional autoregressive root in the nonfundamental representation will be the autoregressive parameter of the single filtering error, $f_{t}=k_{t}-\widehat{k}_{t}$.
} 
However, some forms of market incompleteness can imply that the marketconsistent information set is non-invertible. Our third example can be derived from the framework of Graham and Wright (2010), who analyse an incomplete markets version of the stochastic growth model in which there is a unified market for capital, but households earn a wage with an idiosyncratic component. As a result their (agent-specific) market-consistent information set does not contain the history of the aggregate wage. In a limiting case in which each household's individual wage is an arbitrarily poor signal of the aggregate wage, it is possible to show that the (common) information set on the aggregate economy consists only of the history of the return on capital and hence is non-invertible, as in our third example. ${ }^{33}$

\section{Conclusions}

In this paper we have presented a general method of solving the signal extraction problem in linear rational expectations models, with particular focus on the properties that arise in linearised DSGE models. We allow for endogeneity between forward-looking variables and both measured and state variables. We derive a number of key features of such economies, that relate to the nature of the solution of the Kalman filter, focussing in particular on whether the information set is invertible, i.e. whether it can replicate full information, at least asymptotically.

Our framework has considered a class of models where the signal extraction problem can be represented either as that of a single forward-looking agent, or a set of such agents who share the same information set. There is potentially a much wider class of models in which individual agents, or types of agent, have overlapping, but not common information sets, which typically results a "hierarchy of expectations" (Townsend, 1983, Woodford, 2003) Even in such models, however, the techniques outlined in this paper are still a crucial part of the solution when states are endogenous (see for example, Graham and Wright, 2010); and limiting cases can have an identical form to the symmetric information equilibrium set out in this paper.

We have emphasised the application of our techniques to DSGE models. However most of our results are quite general and in principle applicable in a wide variety of contexts where dynamic optimisation problems involve states that are both endogenous and not directly observable. As such the techniques set out in this paper

\footnotetext{
${ }^{33}$ It is also possible to show that in this limiting case there is a modified law of motion of aggregate capital; but it is still of the same general form as (37). Furthermore, aggregate consumption can be written as the optimising decision of a single representative agent. Details of the derivation of this limiting case are available from the authors.
} 
broaden out further the already wide scope for application of the Kalman filter.

\section{References}

Anderson, D., Moore, J.B., 1979. Optimal Filtering. Dover.

Blanchard, O, Kahn, J.A., 1980. The Solution of Linear Difference Models under Rational Expectations. Econometrica 48 (5), pp.1305-1312.

Bomfim, A,N, 2004. Measurement error in general equilibrium: the aggregate effects of noisy economic indicators. Journal of Monetary Economics 48, pp.505-603.

Campbell, J.Y., 1994. Inspecting the mechanism: an analytical approach to the stochastic growth model. Journal of Monetary Economics 33, pp.463-506.

Collard, F., Dellas, H., 2010. Monetary Misperceptions, Output, and Inflation Dynamics. Journal of Money, Credit and Banking,42(2-3), pp. 483-502, 03.

Fernandez-Villaverde, J., Rubio-Ramirez, F., Sargent, T., Watson, M., 2007. ABCs (and Ds) of Understanding VARs. American Economic Review, pp. 1021-1026.

Graham, L., Wright, S., 2010. Information, heterogeneity and market incompleteness. Journal of Monetary Economics, 57, pp 164 - 174.

Hansen, L., and Sargent, T., 2005. Recursive Models of Dynamic Linear Economies. Monograph.

Harvey, A., 1981. Time Series Models. Philip Allan, London.

Harvey, A., 1989. Forecasting, Structural Time Series Models and the Kalman Filter. Cambridge University Press.

Hamilton, J., 1994. Time Series Analysis. Princeton University Press.

Keen, B.D., 2010. The Signal Extraction Problem Revisited: A Note on Its Impact on a Model of Monetary Policy. Macroeconomic Dynamics, 14(3), pp. 405-426.

Lippi, M., Reichlin, L., 1994. VAR analysis, nonfundamental representations, Blaschke matrices. Journal of Econometrics, 63 pp 307-325

Lorenzoni, G., 2009. A Theory of Demand Shocks". American Economic Review, $99(5), 2050-84$. 
McCallum, B.T., 1998. Solutions to linear rational expectations models: a compact exposition. Economics Letters 61, pp.143-147.

Mehra, R., Prescott, E.C., 1980. Recursive Competitive Equilibrium: The Case of Homogeneous Households. Econometrica 48 (6), pp.1365-1379.

Muth, J.F., 1961. Rational Expectations and the Theory of Price Movements. Econometrica 29 (3), pp.315-335.

Nimark, K., 2007. Dynamic Higher Order Expectations. Working Paper.

Pearlman, J., Currie, D., Levine P.,1986. Rational Expectations Models with Partial Information". Economic Modeling 3, pp 90-105.

Pearlman, J., 1992. Reputational and nonreputational policies under partial information. Journal of Economic Dynamics and Control 16 (2), pp.339-357.

Porapakkarm, P., Young, E.R., 2008. Information Heterogeneity in the Macroeconomy. Working paper.

Sims, C.A., 2003. Implications of rational inattention. Journal of Monetary Economics, 50 (3), pp. $665-690$.

Söderström, T 1994. Discrete-Time Stochastic Systems. Prentice Hall, London, New York.

Svennson, L.E.O., Woodford, M., 2003. Indicator variables and optimal policy. Journal of Monetary Economics 50 (3), pp.691-720.

Svennson, L.E.O., Woodford, M., 2004. Indicator variables for optimal policy under asymmetric information. Journal of Economic Dynamics and Control 28 (4), pp.661690.

Townsend, R.M., 1983. Forecasting the Forecasts of Others. Journal of Political Economy 91 (4), pp 546-588.

Whittle, P., 1983. Prediction and Regulation by Linear Least-Squares Methods. University of Minnesota Press.

Woodford, M, 2003. Imperfect Common Knowledge and the Effects of Monetary Policy, in Aghion P., Frydman, R., Stiglitz, J., Woodford, M., (Eds.), Knowledge, Information, and Expectations in Modern Macroeconomics: In Honour of Edmund S. Phelps, Princeton University Press. 
Supplementary material for

"Invertible and non-invertible information sets in linear rational expectations models"

by Brad Baxter, Liam Graham ${ }^{1}$ and Stephen Wright.

\section{A Derivation of Equations (7) and (8)}

We first stack equations (2) on top of (3) and (6) to derive the law of motion for the state variables, $\xi_{t}=\left[\begin{array}{lll}k_{t} & z_{t} & w_{t}\end{array}\right]^{\prime}$, and respecify (5) accordingly,

$$
\begin{gathered}
\xi_{t+1}=D_{\xi \xi} \xi_{t}+D_{\xi y} y_{t}+v_{t+1} \\
i_{t}=D_{i \xi} \xi_{t}+D_{i y} y_{t}
\end{gathered}
$$

where $v_{t}=\left[\begin{array}{lll}0 & \zeta_{t} & \omega_{t}\end{array}\right]^{\prime}=F_{u} u_{t}$, as in (10).

$$
D_{\xi \xi}=\left[\begin{array}{ccc}
B_{k k} & B_{k z} & 0 \\
0 & B_{z z} & 0 \\
0 & 0 & B_{w w}
\end{array}\right] ; D_{\xi y}=\left[\begin{array}{c}
B_{k y} \\
0 \\
0
\end{array}\right] ; D_{i \xi}=\left[\begin{array}{ccc}
C_{i k} & C_{i z} & C_{i w}
\end{array}\right] ; \quad D_{i y}=C_{i y}
$$

We next partition $y_{t}$ as $y_{t}=\left[\begin{array}{ll}c_{t}^{\prime} & x_{t}^{\prime}\end{array}\right]^{\prime}$ and express (1) conformably as: ${ }^{2}$

$$
\left[\begin{array}{cc}
A_{c c} & A_{c x} \\
0 & 0
\end{array}\right] E_{t}\left[\begin{array}{l}
c_{t+1} \\
x_{t+1}
\end{array}\right]=\left[\begin{array}{cc}
B_{c c} & B_{c x} \\
B_{x c} & B_{x x}
\end{array}\right]\left[\begin{array}{l}
c_{t} \\
x_{t}
\end{array}\right]+\left[\begin{array}{c}
B_{c \xi} \\
B_{x \xi}
\end{array}\right] \xi_{t}
$$

where the first block of equations are expectational difference equations and thus represent forward-looking variables such as consumption or policy variables The second block of equations represent purely static relationships (for example, intratemporal optimality conditions, production functions, identities, etc.) that can

\footnotetext{
${ }^{1}$ Corresponding author: Department of Economics, University College London, Gower Street, London WC1E 6BT, UK. Liam.Graham@ucl.ac.uk. Phone: +44 207679 5850. Fax: +44 207916 2775 .

${ }^{2}$ This form for $A_{y y}$ will usually follow naturally from the structure of the model, but as long as $A_{y y}$ is singular (if it is not, then the vector $x_{t}$ will be empty) this structure can always be achieved by an appropriate linear re-weighting of the elements of $y_{t}$. The sub-matrix $A_{c x}$ may also in principle contain columns of zeros.
} 
be substituted out. Using these, assuming $B_{x x}^{-1}$ exists $^{3}$ we can substitute out using

$$
x_{t}=-B_{x x}^{-1}\left[B_{x c} c_{t}+B_{x \xi} \xi_{t}\right]=D_{x c} c_{t}+D_{x \xi} \xi_{t}
$$

and write the state and measurement equation in their final form in the main text as

$$
\begin{gathered}
\xi_{t+1}=F_{\xi} \xi_{t}+F_{c} c_{t}+v_{t+1} \\
i_{t}=H_{\xi}^{\prime} \xi_{t}+H_{c} c_{t}
\end{gathered}
$$

where $D_{\xi y}=\left[\begin{array}{cc}D_{\xi c} & D_{\xi x}\end{array}\right], D_{i y}=\left[\begin{array}{ll}D_{i c} & D_{i x}\end{array}\right]$, etc., and

$$
\begin{aligned}
& F_{\xi}=D_{\xi \xi}+D_{\xi x} D_{x \xi} ; \quad F_{c}=D_{\xi c}+D_{\xi x} D_{x c} \\
& H_{\xi}^{\prime}=D_{i \xi}+D_{i x} D_{x \xi} \quad H_{c}=D_{i c}+D_{i x} D_{x c}
\end{aligned}
$$

Note that the substitutions involved in deriving (7) and (8) are by no means innocuous in informational terms.

First, even static relationships may require informational assumptions. Since they may involve linear combinations of state variables it may be of considerable importance whether these combinations, or the elements of $x_{t}$ themselves, are in the information set $I_{t}$. The form of the measurement equation allows for the possibility that elements of $x_{t}$ may be observable, whether directly or indirectly, but there are interesting cases where they are not. 4

The nature of the expectational difference equations satisfied by $c_{t}$, the forwardlooking variables, may also have important informational implications. While this framework can in principle accommodate any structure to the top block of equations in (A.3), certain structures may require assumptions about the nature of the information set. Thus if we substitute out for $x_{t}$ using (A.4) and (7) and use (16) we can write the top block, applying the law of iterated expectations, as

$$
\left\{A_{c c} \eta^{\prime}+A_{c x}\left(D_{x c} \eta^{\prime}+D_{x \xi}\right)\right\} G \widehat{\xi}_{t}=\left\{B_{c c}+B_{c x} D_{x c}\right\} \eta^{\prime} \widehat{\xi}_{t}+\left\{B_{c \xi}+B_{c x} D_{x \xi}\right\} \xi_{t}
$$

which depends on $\xi_{t}$ as well as $\widehat{\xi}_{t}$. For such a formulation to be informationally feasible in this precise form, the linear combination of states given by $\left\{B_{c \xi}+B_{c x} D_{x \xi}\right\} \xi_{t}$ must be observable, and therefore should also be an element of $i_{t}$. In principle this may significantly alter the information set and hence the nature of the filtering

\footnotetext{
${ }^{3}$ The case where $B_{x x}^{-1}$ does not exist implies that some elements of $x_{t}$ can be expressed as linear combinations of other elements, and can thus be trivially dealt with by substitution.

${ }^{4}$ In Graham and Wright (2010), for example, the aggregate wage is an element of $x_{t}$ that is not observable, given heterogeneous labour and incomplete markets.
} 
problem (although the rationale for this combination being observable should be justifiable). If this linear combination is indeed observable, then (from Corollary 4) efficiency of the state estimates requires that they satisfy the adding up constraint $\left\{B_{c \xi}+B_{c x} D_{x \xi}\right\} \xi_{t}=\left\{B_{c \xi}+B_{c x} D_{x \xi}\right\} \widehat{\xi}_{t}$ thus allowing the top block to be written entirely in terms of state estimates, as

$$
\left\{A_{c c} \eta^{\prime}+A_{c x}\left(D_{x c} \eta^{\prime}+D_{x \xi}\right)\right\} \widehat{\xi}_{t}=\left[\left\{B_{c c}+B_{c x} D_{x c}\right\} \eta^{\prime}+B_{c \xi}+B_{c x} D_{x \xi}\right] \widehat{\xi}_{t}
$$

which results in an undetermined coefficients problem identical to that under full information. Note also that the nature of the undetermined coefficients problem is unchanged if this linear combination of states is not observable, but is replaced by the same combination of state estimates.

This issue does not, of course, arise if, as in many contexts (for example consumption Euler equations) $B_{c x}$ and $B_{c \xi}$ are zero.

\section{B Proof of Proposition 1}

For completeness, and because some elements of the proof are of relevance to later analysis, we present a full proof of the proposition. Most strictly algebraic elements of the proof are however to be found in the existing literature on optimal control. For example, Söderström (1994, Ch. 6) derives the solution to a filtering problem with what we refer to here as "dynamic endogeneity" (i.e. $F_{c} \neq 0$ ). His solution is identical to ours if we set $H_{c}=0$, once we allow for differences in notation; he does not however explicitly draw out the link with the "parallel problem". We also show in Section B.3 of this appendix that our approach yields identical answers to those derived in Svensson and Woodford (2003), despite their rather unusual statement of the filtering process.

We assume that in some period $t-1$ initial estimates of the states $\xi_{t}$ and $P_{t}$ are available, that must satisfy $E_{t-1} \widehat{\xi}_{t}=E_{t-1} \xi_{t}$ by the law of iterated expectations, given the definition of $\widehat{\xi}_{t}$. This condition will always be satisfied if, at $t=0, E_{0} \widehat{\xi}_{1}=E_{0} \xi_{1}$.

\section{B.1 Forecasting $i_{t}$}

Using (8)

$$
E_{t-1} i_{t}=H_{\xi}^{\prime} E_{t-1} \xi_{t}+H_{c} \eta^{\prime} E_{t-1} \widehat{\xi}_{t}=\left(H_{\xi}^{\prime}+H_{c} \eta^{\prime}\right) E_{t-1} \widehat{\xi}_{t}
$$


where the second expression follows by the law of iterated expectations. The error of this forecast is, using (24), (5) and (16)

$$
\begin{aligned}
\varepsilon_{t} & \equiv i_{t}-E_{t-1} i_{t}=H_{\xi}^{\prime}\left[\xi_{t}-E_{t-1} \widehat{\xi}_{t}\right]+H_{c} \eta^{\prime}\left[\widehat{\xi}_{t}-E_{t-1} \widehat{\xi}_{t}\right] \\
& =H_{\xi}^{\prime}\left[\xi_{t}-E_{t-1} \xi_{t}\right]+H_{c} \eta^{\prime}\left[\widehat{\xi}_{t}-E_{t-1} \widehat{\xi}_{t}\right]
\end{aligned}
$$

where the second line follows from the definition of $\widehat{\xi}_{t}$, after (16), by applying the Law of Iterated Expectations. We then treat (22), the process for the estimated states, as a conjectured solution to the filtering process. Conditional upon this conjectured solution we have

$$
\varepsilon_{t}=H_{\xi}^{\prime}\left[\xi_{t}-E_{t-1} \xi_{t}\right]+H_{c} \eta^{\prime} \beta_{t} \varepsilon_{t}
$$

hence

$$
\varepsilon_{t}=J_{t}^{\prime}\left[\xi_{t}-E_{t-1} \xi_{t}\right]
$$

where

$$
J_{t}^{\prime}=\left[I_{n}-H_{c} \eta^{\prime} \beta_{t}\right]^{-1} H_{\xi}^{\prime}
$$

Thus we have, using (B.3) and (18)

$$
E\left[\varepsilon_{t} \varepsilon_{t}^{\prime}\right]=J_{t}^{\prime} E\left[\left(\xi_{t}-E_{t-1} \xi_{t}\right)\left(\xi_{t}-E_{t-1} \xi_{t}\right)^{\prime}\right] J_{t}=J_{t}^{\prime} P_{t} J_{t}
$$

\section{B.2 Deriving $\beta_{t}, M_{t}$ and $P_{t}$.}

Since (conditional upon $\beta_{t}$ and hence $J_{t}$ ) innovations to $i_{t}$ depend only on unobservable errors in forecasting the states, the Kalman Gain matrix, $\beta_{t}$ in the updating equation $(22)$ is

$$
\beta_{t}=\left\{E\left[\left(\xi_{t}-E_{t-1} \xi_{t}\right) \varepsilon_{t}^{\prime}\right]\right\}\left\{E\left[\varepsilon_{t} \varepsilon_{t}^{\prime}\right]\right\}^{-1}
$$

Hence, using (B.3), (18), and (B.5),

$$
\beta_{t}=P_{t} J_{t}\left[J_{t}^{\prime} P_{t} J_{t}\right]^{-1}
$$

and the MSE of the state estimates can be written as

$$
\begin{aligned}
M_{t} & =E\left[\left(\xi_{t}-E_{t-1} \widehat{\xi}_{t}\right)\left(\xi_{t}-E_{t-1} \widehat{\xi}_{t}\right)^{\prime}\right]-\beta_{t} E\left[\varepsilon_{t}\left(\xi_{t}-E_{t-1} \widehat{\xi}_{t}\right)^{\prime}\right] \\
& =P_{t}-\beta_{t} J_{t}^{\prime} P_{t}=\left[I_{r}-\beta_{t} J_{t}^{\prime}\right] P_{t}
\end{aligned}
$$


however these do not yet constitute closed form solutions since, via (B.4), $J_{t}$ depends on $\beta_{t}$.

To show that the recursion for $P_{t+1}$ is $F_{c}$-independent, note that the forecast error in predicting the states in period $t+1$ is, using (14), and (7),

$$
\begin{aligned}
\xi_{t+1}-E_{t} \widehat{\xi}_{t+1} & =F_{\xi} \xi_{t}+F_{c} \eta^{\prime} \widehat{\xi}_{t}+v_{t+1}-\left(F_{\xi}+F_{c} \eta^{\prime}\right) \widehat{\xi}_{t} \\
& =F_{\xi}\left(\xi_{t}-\widehat{\xi}_{t}\right)+v_{t+1}
\end{aligned}
$$

and is thus independent of $F_{c}$. Hence, using the orthogonality assumptions and (17),

$$
P_{t+1}=F_{\xi} M_{t} F_{\xi}^{\prime}+Q
$$

To show that $P_{t+1}$ and $M_{t}$ are $H_{c}$-independent, let

$$
J_{t}^{\prime}=K_{t}^{-1} H_{\xi}^{\prime}
$$

where $K_{t}$ is the (as yet unknown) matrix that satisfies

$$
\begin{gathered}
K_{t}=\left(I_{n}-H_{c} \eta^{\prime} \beta_{t}\right) . \\
\Rightarrow\left(J_{t}^{\prime} P_{t} J_{t}\right)^{-1}=\left(K_{t}^{-1} H_{\xi}^{\prime} P_{t} H_{\xi}\left(K_{t}^{-1}\right)^{\prime}\right)^{-1}=K_{t}^{\prime}\left(H_{\xi}^{\prime} P_{t} H_{\xi}\right)^{-1} K_{t}
\end{gathered}
$$

and hence, substituting (B.11) and (B.13) into (B.7), we obtain

$$
\beta_{t}=P_{t} H_{\xi}\left(H_{\xi}^{\prime} P_{t} H_{\xi}\right)^{-1} K_{t}
$$

and thus

$$
\begin{aligned}
\beta_{t} J_{t}^{\prime} & =P_{t} H_{\xi}\left(K_{t}^{-1}\right)^{\prime} K_{t}^{\prime}\left(H_{\xi}^{\prime} P_{t} H_{\xi}\right)^{-1} K_{t} K_{t}^{-1} H_{\xi}^{\prime} \\
& =P_{t} H_{\xi}\left(H_{\xi}^{\prime} P_{t} H_{\xi}\right)^{-1} H_{\xi}^{\prime} .
\end{aligned}
$$

We thus have

$$
\begin{gathered}
M_{t}=\left(I_{r}-P_{t} H_{\xi}\left(H_{\xi}^{\prime} P_{t} H_{\xi}\right)^{-1} H_{\xi}^{\prime}\right) P_{t}, \\
P_{t+1}=F_{\xi}\left(I_{r}-P_{t} H_{\xi}\left(H_{\xi}^{\prime} P_{t} H_{\xi}\right)^{-1} H_{\xi}^{\prime}\right) P_{t} F_{\xi}^{\prime}+Q,
\end{gathered}
$$

Thus the recursions for $M_{t}$ and $P_{t}$ do not depend on $F_{c}$ or $K_{t}$ (and hence $H_{c}$ ) and can thus be derived by setting $H_{c}=F_{c}=0$ as in the parallel problem. If we define $\widetilde{\beta}_{t}$ as in (21) then the above formulae are identical to (19) and (20) in Proposition 
1. We also have, using (B.15)

$$
\beta_{t} J_{t}^{\prime}=\tilde{\beta}_{t} H_{\xi}^{\prime}
$$

Finally we need to obtain an expression for $J_{t}$ itself, and hence for $\beta_{t}$. Equations (B.4) and (B.7) imply the seemingly nonlinear equation

$$
J_{t}^{\prime}=\left(I_{n}-H_{c} \eta^{\prime} P_{t} J_{t}\left(J_{t} P_{t} J_{t}^{-1}\right)\right)^{-1} H_{\xi}^{\prime}
$$

However, using (B.11) and (B.14), we obtain

$$
\begin{gathered}
K_{t}^{-1} H_{\xi}^{\prime}=J_{t}^{\prime}=\left(I_{n}-H_{c} \eta^{\prime} P_{t} H_{\xi}\left(H_{\xi}^{\prime} P_{t} H_{\xi}\right)^{-1} K_{t}\right)^{-1} H_{\xi}^{\prime}, \\
\Rightarrow K_{t}^{-1} H_{\xi}^{\prime}-H_{c} \eta^{\prime} P_{t} H_{\xi}\left(H_{\xi}^{\prime} P_{t} H_{\xi}\right)^{-1} H_{\xi}^{\prime}=H_{\xi}^{\prime}
\end{gathered}
$$

Recalling (B.11) once again, we find

$$
\begin{gathered}
J_{t}^{\prime}=H_{\xi}^{\prime}+H_{c} \eta^{\prime} P_{t} H_{\xi}\left(H_{\xi}^{\prime} P_{t} H_{\xi}\right)^{-1} H_{\xi}^{\prime} \\
=\left(I_{n}+H_{c} \eta^{\prime} P_{t} H_{\xi}\left(H_{\xi}^{\prime} P_{t} H_{\xi}\right)^{-1}\right) H_{\xi}^{\prime} \\
\Rightarrow K_{t}=\left[I_{n}+H_{c} \eta^{\prime} P_{t} H_{\xi}\left(H_{\xi}^{\prime} P_{t} H_{\xi}\right)^{-1}\right]^{-1}
\end{gathered}
$$

Using (21), these can be expressed as

$$
\begin{aligned}
& J_{t}^{\prime}=\left(I_{n}+H_{c} \eta^{\prime} \widetilde{\beta}_{t}\right) H_{\xi}^{\prime} \\
& K_{t}=\left(I_{n}+H_{c} \eta^{\prime} \widetilde{\beta}_{t}\right)^{-1}
\end{aligned}
$$

which, after substituting from (B.24) into (B.14) gives (23), completing the proof of Proposition 1 .

\section{B.3 Comparison with Svensson and Woodford (2003) and Pearlman et al. (1986)}

Svensson and Woodford (2003) have a structural model which in reduced form is extremely close to ours. Their equations (15) and (16) correspond directly to our state and measurement equations (7) and (8), after substituting from (16). Using their notation their equation (22) is

$$
X_{t \mid t}=X_{t \mid t-1}+K\left[L\left(X_{t}-X_{t \mid t-1}\right)+v_{t}\right]
$$


where, $X_{t \mid t}$ in their notation corresponds to $\widehat{\xi}_{t}$ in ours, and $Z_{t}$ to our $i_{t}$. They then assert that this allows them to identify $K$ as " (one form of) the Kalman Gain Matrix" (which they assume, without proof, will converge to a fixed matrix). However, by the usual convention in the literature the Kalman gain updates in response to a forecast error. The square bracketed expression is not a forecast error. Using their (16), the true forecast error in their framework is

$$
Z_{t}-E_{t-1} Z_{t}=L\left(X_{t}-X_{t \mid t-1}\right)+M\left(X_{t \mid t}-X_{t \mid t-1}\right)+v_{t}
$$

where the endogeneity of the measured variables to the response of the estimated states is evident.

However, it turns out that, despite the somewhat unusual basis for their derivation, their final result is in fact identical to our own. If we re-express their (22) in our own notation (apart from the matrix $K$ ), it becomes

$$
\widehat{\xi}_{t}-E_{t-1} \widehat{\xi}_{t}=K\left[H_{\xi}^{\prime}\left(\xi_{t}-E_{t-1} \widehat{\xi}_{t}\right)\right]
$$

whereas we show that, in our notation, from (B.3), after substituting from the endogenous response of $c_{t}$, and assuming convergence, the updating rule in response to the forecast error in the measured variables is given by

$$
\widehat{\xi}_{t}-E_{t-1} \widehat{\xi}_{t}=\beta \varepsilon_{t}=\beta J^{\prime}\left(\xi_{t}-E_{t-1} \widehat{\xi}_{t}\right)
$$

But using their equations (24) and (25) (noting that we absorb the covariance matrix of measurement errors into $Q$, and hence $P$ ), their derivation implies, in our notation,

$$
K=\widetilde{\beta}
$$

thus in our notation $K$ is identical to the Kalman gain matrix in the parallel, rather than the actual problem. But, from (B.18), we have $\beta J^{\prime}=\widetilde{\beta} H_{\xi}^{\prime}$, hence

$$
\widehat{\xi}_{t}-E_{t-1} \widehat{\xi}_{t}=\beta J^{\prime}\left(\xi_{t}-E_{t-1} \widehat{\xi}_{t}\right)=\widetilde{\beta} H_{\xi}^{\prime}\left(\xi_{t}-E_{t-1} \widehat{\xi}_{t}\right)
$$

thus Svensson and Woodford's updating rule is in fact identical to our own. An equivalent updating rule is also given in Pearlman et al. (1986) equation (39). However, neither of these papers note the equivalence of $M_{t}$ and $P_{t+1}$ in the parallel problem, nor do they derive convergence conditions. 


\section{Proof of Proposition 2 and Corollary 2.}

\section{C.1 Proof of Proposition 2}

Since $\beta_{t}$ and $M_{t}$ can both be expressed in terms of $P_{t}$ and structural parameters a necessary and sufficient condition for convergence of all three matrices to a unique steady state is convergence of $P_{t}$ to a unique steady state. Since $P_{t}$ can be derived from the the parallel problem of Proposition 1 in which the states are exogenous we only need be concerned with the stability properties of that problem. Anderson and Moore (1979, pp. 77-81) provide a proof of a unique stable steady state given controllability and detectability as defined in the main text for any invertible $P_{0}$.

\section{C.2 Proof of Corollary 2}

We first restate (19), writing $F \equiv F_{\xi}, H \equiv H_{\xi}$ in this section, for brevity, as

$$
P_{t+1}=F\left(I_{r}-P_{t} H\left(H^{\prime} P_{t} H\right)^{-1} H^{\prime}\right) P_{t} F^{\prime}+Q .
$$

In other words, we are iterating the function $g: \mathbb{P}_{r} \rightarrow \mathbb{P}_{r}$, where $\mathbb{P}_{r}$ denotes the set of all non-negative definite symmetric, real $r \times r$ matrices, and

$$
g\left(P_{t}\right)=F\left(I_{r}-P_{t} H\left(H^{\prime} P_{t} H\right)^{-1} H^{\prime}\right) P_{t} F^{\prime}+Q, \quad P_{t} \in \mathbb{P}_{r}
$$

If the conditions set by Proposition 2 are satisfied, then this iteration is stable around a unique fixed point $P$

We first note a convenient simplification. Let

$$
\widehat{F}\left(P_{t}\right)=F\left(I_{r}-P_{t} H\left(H^{\prime} P_{t} H\right)^{-1} H^{\prime}\right)=F\left(I_{r}-\widetilde{\beta}\left(P_{t}\right) H^{\prime}\right)
$$

(where the second expression uses (21)) then:

Lemma 1 The function $g: \mathbb{P}_{r} \rightarrow \mathbb{P}_{r}$ defined by (C.2) can be expressed, using (C.3), in the symmetric form

$$
g\left(P_{t}\right)=\widehat{F}\left(P_{t}\right) P \widehat{F}\left(P_{t}\right)^{\prime}+Q
$$

Proof. Using (C.3), we have

$$
\begin{gathered}
g\left(P_{t}\right)=\widehat{F}\left(P_{t}\right) P_{t} F^{\prime}+Q \\
\widehat{F} P \widehat{F}^{\prime}=\widehat{F} P F^{\prime}-F\left(I_{r}-\widetilde{\beta} H^{\prime}\right) P H \widetilde{\beta}^{\prime} F^{\prime}
\end{gathered}
$$


but

$$
\left(I_{r}-\widetilde{\beta} H^{\prime}\right) P H \widetilde{\beta}^{\prime}=P H \widetilde{\beta}^{\prime}-\widetilde{\beta} H^{\prime} P H \widetilde{\beta}^{\prime}=P H \widetilde{\beta}^{\prime}-P H\left(H^{\prime} P H\right)^{-1} H^{\prime} P H \widetilde{\beta}^{\prime}=0
$$

As is usual in the analysis of fixed point iteration, we must calculate the (Fréchet) derivative of $g$ at the fixed point $P$.

Lemma 2 If $E \in \mathbb{P}_{r}$, then, letting $\widehat{F}_{P}=\widehat{F}(P)$

$$
g(P+E)=g(P)+\widehat{F}_{P} E \widehat{F}_{P}^{\prime}+O\left(E^{2}\right)
$$

Thus if we let $D g_{P}$ denote the Fréchet derivative of the matrix function $g$ at the point $P \in \mathbb{P}_{r}$, then

$$
D g_{P}(E)=\widehat{F}_{P} E \widehat{F}_{P}^{\prime} \quad E \in \mathbb{P}_{r}
$$

Proof. We have, using (C.2)

$$
\begin{aligned}
& g(P+E) \\
& =F(P+E) F^{\prime}-F(P+E) H\left(H^{\prime} P H+H^{\prime} E H\right)^{-1} H^{\prime}(P+E) F^{\prime}+Q \\
& =F(P+E) F^{\prime}-F(P+E) H\left[\left(H^{\prime} P H\right)\left(I+\left(H^{\prime} P H\right)^{-1}\left(H^{\prime} E H\right)\right)\right]^{-1} H^{\prime}(P+E) F^{\prime}+Q \\
& =\ldots-F(P+E) H\left[I-\left(H^{\prime} P H\right)^{-1}\left(H^{\prime} E H\right)\right]\left(H^{\prime} P H\right)^{-1} H^{\prime}(P+E) F^{\prime}+Q+O\left(E^{2}\right) \\
& =g(P)+\widehat{F}_{P} E \widehat{F}_{P}^{\prime}+O\left(E^{2}\right) .
\end{aligned}
$$

It is useful to restate (C.8) and (C.9) in Kronecker product notation, as

$$
\operatorname{vec}(g(P+E))=\operatorname{vec}(g(P))+\widehat{F}_{P} \otimes \widehat{F}_{P} \operatorname{vec}(E)+O\left(\operatorname{vec}\left(E^{2}\right)\right)
$$

hence, in this form the Fréchet derivative is (using (C.3))

$$
D g_{P}=\widehat{F}_{P} \otimes \widehat{F}_{P}=F\left(I_{r}-\widetilde{\beta}(P) H^{\prime}\right) \otimes F\left(I_{r}-\widetilde{\beta}(P) H^{\prime}\right)
$$

and thus as a corollary of Proposition 2, stability of the steady state implies that the matrix $F_{\xi}\left(I_{r}-\widetilde{\beta}(P) H_{\xi}^{\prime}\right)$ must have eigenvalues with real parts strictly less than one in absolute value. Since products of matrices have common non-zero eigenvalues irrespective of order of multiplication this condition must also apply to the matrix $\left(I_{r}-\widetilde{\beta}(P) H_{\xi}^{\prime}\right) F_{\xi}$. The proof of Corollary 4 shows that this matrix will be of rank $\leq r-n$. 


\section{C.3 Proof of Corollary 1.}

From the autoregressive representation of the estimated states in (22) it is evident that they have the same autoregressive form as the true states under full information in (13). To derive the implied innovation covariance matrix we have, using (24), (B.3), (B.5) and (B.18),

$$
\begin{aligned}
E\left[\left(\widehat{\xi}_{t+1}-E_{t} \widehat{\xi}_{t+1}\right)\left(\widehat{\xi}_{t+1}-E_{t} \widehat{\xi}_{t+1}\right)^{\prime}\right] & =\beta E\left(\varepsilon_{t+1} \varepsilon_{t+1}^{\prime}\right) \beta^{\prime} \\
& =\beta J^{\prime} P J \beta^{\prime}=\widetilde{\beta} H_{\xi}^{\prime} P H_{\xi} \widetilde{\beta}^{\prime}
\end{aligned}
$$

but hence, using (19), (21), (20), and exploiting symmetry of $P$ and $M$

$$
\begin{aligned}
E\left[\left(\widehat{\xi}_{t+1}-E_{t} \widehat{\xi}_{t+1}\right)\left(\widehat{\xi}_{t+1}-E_{t} \widehat{\xi}_{t+1}\right)^{\prime}\right] & =P H_{\xi}\left[H_{\xi}^{\prime} P H_{\xi}\right]^{-1} P H_{\xi} \widetilde{\beta}^{\prime} \\
& =P H_{\xi} \widetilde{\beta}^{\prime}=\left(\beta H^{\prime} P\right)^{\prime} \\
= & (P-M)^{\prime}=Q+F_{\xi} M F_{\xi}^{\prime}-M
\end{aligned}
$$

\section{Derivation of joint process for $\xi_{t}$ and $f_{t}$ in (27) and proofs of corollaries 3 to 5}

\section{D.1 Derivation of (27).}

Using (7), (16) and (26) we have

$$
\begin{aligned}
\xi_{t+1} & =F_{\xi} \xi_{t}+F_{c} \eta^{\prime} \widehat{\xi}_{t}+v_{t+1}=\left(F_{\xi}+F_{c} \eta^{\prime}\right) \xi_{t}-F_{c} \eta^{\prime} f_{t}+v_{t+1} \\
& =G \xi_{t}-F_{c} \eta^{\prime} f_{t}+v_{t+1}
\end{aligned}
$$

For the estimated states we have, using (22), the definition of $G$ in (13), (B.3) and (7)

$$
\begin{aligned}
\widehat{\xi}_{t+1} & =G \widehat{\xi}_{t}+\beta_{t} \varepsilon_{t+1}=G \widehat{\xi}_{t}+\beta_{t} J_{t}^{\prime}\left[\xi_{t+1}-G \widehat{\xi}_{t}\right] \\
& =G \widehat{\xi}_{t}+\beta_{t} J_{t}^{\prime}\left[\xi_{t+1}-\left(F_{\xi}+F_{c} \eta^{\prime}\right) \widehat{\xi}_{t}\right] \\
& =G \widehat{\xi}_{t}+\beta_{t} J_{t}^{\prime}\left[\xi_{t+1}-F_{\xi} \xi_{t}-F_{c} \eta^{\hat{\xi}_{t}}+F_{\xi}\left(\xi_{t}-\widehat{\xi}_{t}\right)\right] \\
& =G \widehat{\xi}_{t}+\beta_{t} J_{t}^{\prime}\left[v_{t+1}+F_{\xi} f_{t}\right]
\end{aligned}
$$


hence, using (14), (B.18) and (10),

$$
\begin{aligned}
f_{t+1} & =\left(G-F_{c} \eta^{\prime}-\beta_{t} J_{t}^{\prime} F_{\xi}\right) f_{t}+\left(I-\beta_{t} J_{t}^{\prime}\right) v_{t+1} \\
& =\left[I-\beta_{t} J_{t}^{\prime}\right] F_{\xi} f_{t}+\left(I-\beta_{t} J_{t}^{\prime}\right) v_{t+1} \\
& =\left[I-\widetilde{\beta}_{t} H_{\xi}^{\prime}\right] F_{\xi} f_{t}+\left(I-\widetilde{\beta}_{t} H_{\xi}^{\prime}\right) F_{u} u_{t+1}
\end{aligned}
$$

Stacking (D.1) above (D.6) gives the joint process for $\xi_{t+1}$ and $f_{t+1}$ in $(27)$.

\section{D.2 Proof of Corollary 3}

Since the filtering error process $f_{t}$ is block recursive we can write the top block of equations as

$$
\begin{aligned}
\xi_{t+1} & =[I-G L]^{-1} v_{t+1}-[I-G L]^{-1} F_{c} \eta^{\prime} f_{t}=\xi_{t+1}^{*}-[I-G L]^{-1} F_{c} \eta^{\prime} f_{t} \\
& =\xi_{t+1}^{*}-[I-G L]^{-1} F_{c} \eta^{\prime}\left[I-\left(I-\widetilde{\beta}_{t} H_{\xi}^{\prime}\right) F_{\xi} L\right]^{-1}\left(I-\widetilde{\beta}_{t} H_{\xi}^{\prime}\right) F_{u} u(\mathrm{D} .8)
\end{aligned}
$$

where $\xi_{t+1}^{*}$, the full information state process, is as given by (13), and the last line uses the lower block of (27). The incomplete information states are thus equal to the full information states plus a lag polynomial in the filtering error (itself a lag polynomial in the underlying shocks, $u_{t}$ ). Since the filtering errors have a limiting stationary distribution (from Corollary 2) and the full information process is nonexplosive (from Assumptions 1 and 2) the incomplete information process is also non-explosive. Since there may be permanent productivity or other shocks, $G$ may have unit eigenvalues, implying permanent effects of these shocks. But permanent effects will only arise with respect to rows of $v_{t}$ for which the relevant rows of $F_{c}$ are, by Assumption 1, zero (the shock processes are exogenous). Hence filtering error will only cause transitory deviations from the full information outcome, proving the corollary.

\section{D.3 Proof of Corollary 4}

Using (21) we have

$$
H_{\xi}^{\prime} \widetilde{\beta}_{t}=H_{\xi}^{\prime} P_{t} H_{\xi}\left(H_{\xi}^{\prime} P_{t} H_{\xi}\right)^{-1}=I_{n}
$$

If we pre-multiply (D.6) by $H_{\xi}^{\prime}$ and use (D.9) we have

$$
H_{\xi}^{\prime} f_{t+1}=H_{\xi}^{\prime}\left[I-\widetilde{\beta}_{t} H_{\xi}^{\prime}\right] F_{\xi} f_{t}+H_{\xi}^{\prime}\left(I-\widetilde{\beta}_{t} H_{\xi}^{\prime}\right) F_{u} u_{t+1}=0
$$


thus proving Corollary 4 , and at the same time showing that the matrix $\left[I-\widetilde{\beta}_{t} H_{\xi}^{\prime}\right] F_{\xi}$ is of rank $\leq r-n$, completing the proof of Corollary 2 .

\section{D.4 Proof of Corollary 5}

By inspection of (27), if $F_{\xi}$ has explosive eigenvalues in its sub-matrix $F_{k k}$ and $\beta_{k}=$ 0 , then the steady-state matrix $\left(I-\widetilde{\beta} H^{\prime}\right) F_{\xi}$ will also have explosive eigenvalues, which, from Corollary 2, contradicts stability of the recursion for $P_{t+1}$, thus proving Corollary 5

\section{E Proof of Proposition 3}

\section{E.1 Proof of Proposition 3}

Recall that in general $M=\lim _{t \rightarrow \infty} M_{t}$ is, from Proposition 2, the unique stable fixed point of the recursion in equations (19) to (21). We showed in the proof of Corollary 2 that stability of the fixed point requires the general eigenvalue condition

$$
\left|\Lambda_{i}\left(I_{r}-\widetilde{\beta}(P) H_{\xi}^{\prime}\right) F_{\xi}\right|<1 \forall i
$$

which we use in what follows.

\section{E.1.1 $M=0 \Rightarrow$ Conditions 1 to 3 .}

If $M=0$ it follows immediately from (19) that $P=Q$. Further, we can infer the the invertibility of $H_{\xi}^{\prime} Q H=\lim _{t \rightarrow \infty} H_{\xi}^{\prime} P_{t} H$ from the existence of a steady state in Proposition 1, and hence, using (11)

$$
H_{\xi}^{\prime} Q H=H_{\xi}^{\prime} F_{u} S F_{u}^{\prime} H
$$

thus

$$
n=\operatorname{rank}\left(H_{\xi}^{\prime} Q H_{\xi}\right) \leq \min \left(\operatorname{rank}\left(H_{\xi}^{\prime} F_{u}\right), s\right)
$$

hence $s \geq n$. Further, (11) and (20) imply, setting $P=Q$

$$
M=F_{u}\left(S-S\left[F_{u}^{\prime} H_{\xi}\left(H_{\xi}^{\prime} F_{u} S F_{u}^{\prime} H_{\xi}\right)^{-1} H_{\xi} F_{u}\right] S\right) F_{u}^{\prime}
$$

which requires $n \geq s$ for $M=0$. Hence $n=s$ and $H_{\xi}^{\prime} F_{u}$ is invertible. Further for this to be a stable fixed point we require the third condition in the proposition, which satisfies (E.1) setting $P=Q$. 


\section{E.1.2 Conditions 1 to $3 \Rightarrow M=0$.}

By Proposition 2 there exists a unique stable fixed point such that $P_{t} \rightarrow P, M_{t} \rightarrow M$, where, taking limits in (19) and (20), using (21) we have

$$
\begin{aligned}
P & =F_{\xi} M F_{\xi}^{\prime}+Q \\
M & =P-P H_{\xi}\left(H_{\xi}^{\prime} P H_{\xi}\right)^{-1} H_{\xi}^{\prime} P
\end{aligned}
$$

Now $P=Q, M=0$ satisfies (E.3). Given conditions 1 and 2 it also satisfies (E.2), which sets $P=Q$ in (E.4). Given condition 3 this is a stable fixed point.

\section{E.2 Equivalence of Proposition 3 with Fernandez-Villaverde et al. (2007)}

Fernandez-Villaverde et al. (2007) analyse the problem of econometric invertibility: i.e. whether an econometrician can infer true structural shocks and impulse responses from an estimated vector autoregressive representation. It turns out that the conditions in which their "square case" is invertible correspond precisely to those given in Proposition 3. They do not, however, draw out the link with pre-determined variables, nor with the stability of the Kalman filter in the neighbourhood of the fixed point corresponding to full information.

To show the correspondence, Fernandez-Villaverde et al. have

$$
\begin{aligned}
& x_{t+1}=A x_{t}+B w_{t+1} \\
& y_{t+1}=C x_{t}+D w_{t+1}
\end{aligned}
$$

where $x_{t}$ is $n \times 1, y_{t}$ is $k \times 1$ and $w_{t}$ is $m \times 1$. Note that the timing convention in (E.6) is somewhat unusual compared to the standard Kalman filter state space representation, in which the measured variables are normally related to the contemporaneous states (possibly plus noise). ${ }^{5}$ They then assume

$$
\begin{array}{r}
m=k \\
|D| \neq 0
\end{array}
$$

implying

$$
w_{t+1}=D^{-1}\left[y_{t+1}-C x_{t}\right]
$$

\footnotetext{
${ }^{5}$ This is however the same timing convention as used by Whittle (1983) p146.
} 
which gives, after substitution into (E.5),

$$
x_{t+1}=\left[A-B D^{-1} C\right] x_{t}+B D^{-1} y_{t+1}
$$

which gives the third condition (their Condition 1, since the first two conditions are taken as implicit)

$$
\left|\Lambda_{i}\left[A-B D^{-1} C\right]\right|<1
$$

where the $\Lambda_{i}$ are eigenvalues. If satisfied, this means that $x_{t+1}$ can be recovered from the infinite history of $y_{t+1}$ (implicitly, even if $n>m=k$, which will be the case, as we note, if there are predetermined variables).

We have (using (6) and (20)), for the states,

$$
\xi_{t+1}=F_{\xi} \xi_{t}+F_{u} u_{t+1}
$$

which with appropriate relabelling of variables and matrices is precisely the same as (E.5). Our measurement equation can be written as

$$
y_{t+1}=H_{\xi} \xi_{t+1}
$$

which, substituting from (E.12) gives

$$
y_{t+1}=H_{\xi}^{\prime} F_{\xi} \xi_{t}+H_{\xi}^{\prime} F_{u} u_{t+1}
$$

which, with appropriate relabelling (i.e. $C=H_{\xi}^{\prime} F_{\xi}, \quad D=H_{\xi}^{\prime} F_{u}$ ) is identical to (E.6). The relabelling makes it clear that their first two conditions are identical to ours. Our third condition relates to the eigenvalues of

$$
\left[I-\beta(Q) H_{\xi}^{\prime}\right] F_{\xi}
$$

but in Proposition 3 we show that

$$
\beta(Q)=F_{u}\left(H_{\xi}^{\prime} F_{u}\right)^{-1}
$$

i.e. can be derived directly from structural matrices. Substituting the crucial matrix becomes

$$
F_{\xi}-F_{u}\left(H_{\xi}^{\prime} F_{u}\right)^{-1} H_{\xi}^{\prime} F_{\xi}
$$

which given the relabelling can be written as

$$
A-B D^{-1} C
$$




\section{F Proof of Proposition 4}

\section{F.1 Proof of part (a)}

We first exploit the diagonalisation $G=V \Lambda V^{-1}$ used in Assumption 2, which allows us to represent the process for the estimated states (22), conditional upon the Kalman Filter having converged, in the diagonalised autoregressive form

$$
X_{t} \equiv V^{-1} \widehat{\xi}_{t}=\Lambda X_{t-1}+\Gamma \varepsilon_{t}
$$

with $\Gamma=V^{-1} \beta$. Note that this subsumes all invertible cases in which we can write $\widehat{\xi}_{t}=\xi_{t} ; \beta \varepsilon_{t}=F_{u} u_{t}$, hence applies irrespective of the nature of the information set. We assume in what follows, without loss of generality, that the diagonal elements of $\Lambda$, the eigenvalues of $G$, are non-zero and distinct (i.e. that we have a minimal state variable representation). We can then rewrite the measurement equation (8) as

$$
i_{t}=\Psi^{\prime} X_{t}
$$

where, exploiting Corollary $4, \Psi^{\prime}=\left(H_{\xi}^{\prime}+H_{c} \eta^{\prime}\right) V$. Note that we have, using (D.9),

$$
\left(H_{\xi}^{\prime}+H_{c} \eta^{\prime}\right) \beta=\left(H_{\xi}^{\prime}+H_{c} \eta^{\prime}\right) \widetilde{\beta}\left[I+H_{c} \eta^{\prime} \tilde{\beta}\right]^{-1}=\left[I+H_{c} \eta^{\prime} \tilde{\beta}\right]\left[I+H_{c} \eta^{\prime} \tilde{\beta}\right]^{-1}=I
$$

and hence

$$
\Psi^{\prime} \Gamma=I
$$

Partition $\Psi^{\prime}$ and order the elements of $X_{t}$ such that we can write

$$
\Psi^{\prime}=\left[\begin{array}{ll}
\Psi_{1} & \Psi_{2}
\end{array}\right] ; \quad\left|\Psi_{1}\right| \neq 0 \Rightarrow X_{1 t}=\Psi_{1}^{-1}\left[i_{t}-\Psi_{2} X_{2 t}\right]
$$

where $\Psi_{1} \in \mathbb{R}^{n \times n}$ and $\Psi_{2} \in \mathbb{R}^{n \times(r-n)}$, hence, exploiting the property that $\Lambda$ is diagonal,

$$
\begin{aligned}
i_{t} & =\Psi^{\prime}\left\{\left[\begin{array}{cc}
\Lambda_{11} & 0 \\
0 & \Lambda_{22}
\end{array}\right]\left[\begin{array}{c}
\Psi_{1}^{-1}\left[i_{t-1}-\Psi_{2} X_{2 t-1}\right] \\
X_{2 t-1}
\end{array}\right]+\left[\begin{array}{c}
\Gamma_{1} \\
\Gamma_{2}
\end{array}\right] \varepsilon_{t}\right\} \\
& =\left[\begin{array}{ll}
\Psi_{1} & \Psi_{2}
\end{array}\right]\left\{\left[\begin{array}{c}
-\Lambda_{11} \Psi_{1}^{-1} \Psi_{2} \\
\Lambda_{22}
\end{array}\right]\left[I-\Lambda_{22} L\right]^{-1} \Gamma_{2} \varepsilon_{t-1}+\left[\begin{array}{c}
\Lambda_{11} \Psi_{1}^{-1} \\
0
\end{array}\right] i_{t-1}\right\}+\varepsilon_{t} \\
& =\Phi_{1}\left[I-\Lambda_{22} L\right]^{-1} \Gamma_{2} \varepsilon_{t-1}+\Phi_{2} i_{t-1}+\varepsilon_{t}
\end{aligned}
$$


where the last two lines use (F.3), and

$$
\Phi_{1}=\Psi^{\prime}\left[\begin{array}{c}
-\Lambda_{11} \Psi_{1}^{-1} \Psi_{2} \\
\Lambda_{22}
\end{array}\right] ; \quad \Phi_{2}=\Psi_{1} \Lambda_{11} \Psi_{1}^{-1}
$$

Hence we can write

$$
i_{t}=\frac{\Phi_{1} \Lambda_{22}^{*}(L)}{\operatorname{det}\left[I-\Lambda_{22} L\right]} \Gamma_{2} \varepsilon_{t-1}+\Phi_{2} i_{t-1}+\varepsilon_{t}
$$

where $\Lambda_{22}^{*}(L)=\operatorname{adj}\left[I-\Lambda_{22} L\right]$. Note that $\operatorname{det}\left[I-\Lambda_{22} L\right]$ is a scalar polynomial of order $r-n$, and the elements of the (diagonal) matrix polynomial $\Lambda_{22}^{*}(L)$ are scalar polynomials of order $r-n-1 .^{6}$ Hence

$$
\lambda(L) i_{t}=\theta(L) \varepsilon_{t}
$$

where

$$
\begin{aligned}
\lambda(L) & =\operatorname{det}\left[I-\Lambda_{22} L\right]\left(I_{n}-\Phi_{2} L\right) \\
\theta(L) & =\operatorname{det}\left[I-\Lambda_{22} L\right] I_{n}+\Phi_{1} \Lambda_{22}^{*}(L) \Gamma_{2} L
\end{aligned}
$$

which is $\operatorname{aARMA}(n, p, q)$ representation with $p=r-n+1$ and $q=r-n$ with both holding with equality because, as assumed at the outset, the diagonal elements of $\Lambda$ (the eigenvalues of $G$ ) are real and distinct. If this is not the case, but $G$ has $\widetilde{r}<r$ distinct non-zero eigenvalues, the argument goes through replacing $r$ with $\widetilde{r}$ throughout. The values of $p$ and $q$ given in the proposition are the maximal orders of the minimal VARMA representation, in which the both the autoregressive and moving average matrix lag polynomials are in general non-diagonal. If these polynomials are restricted to be diagonal (i.e. the system is a set of univariate ARMA representations with correlated innovations) this is equivalent to setting $n=1$.

\section{F.2 Proof of part (b)}

In the case of instantaneous invertibility we can derive the states using (15), then substitute into (7) giving a vector autoregression for $i_{t}$. with innovations given by $\varepsilon_{t}=\left[H_{\xi}^{\prime}+H_{c} \eta^{\prime}\right] F_{u} u_{t}$, where $\varepsilon_{t}$ and $u_{t}$ are both of dimension $r$. In the case of asymptotic invertibility we have a generalised version of (25), given $H_{c} \neq 0$, of the

\footnotetext{
${ }^{6}$ Letting $\left\{\mu_{i}\right\}_{i=1}^{r-n}$ be the eigenvalues of $\Lambda_{22}$, then $\operatorname{det}\left[I-\Lambda_{22} L\right]=\prod_{i=1}^{r-n}\left(1-\mu_{i} L\right)$, and the $i$ th diagonal element of $\left[I-\Lambda_{22} L\right]^{-1}=\left(1-\mu_{i} L\right)^{-1}$, hence the $i$ th diagonal element of $\Lambda_{22}^{*}(L)$ equals $\prod_{j \neq i}\left(1-\mu_{j} L\right)$, a polynomial of order $r-n-1$.
} 
form

$$
\varepsilon_{t+1}=H_{\xi}^{\prime}\left(\xi_{t+1}-E_{t} \xi_{t+1}\right)+H_{c}\left(c_{t+1}-E_{t} c_{t+1}\right)=\left[H_{\xi}^{\prime}+H_{c} \eta^{\prime}\right] F_{u} u_{t}
$$

where the only difference is that $\varepsilon_{t}$ and $u_{t}$ are in this case both of dimension $n<r$

\section{F.3 Proof of parts (c), (d) and (e).}

We first derive a (non-invertible) VARMA representation in terms of the vector of underlying structural innovations $u_{t}$ by exploiting the structure of the proof of part (a) of the proposition, with a relatively small number of changes.

Redefine $X_{t}$ in (F.1) to be the minimal state vector for the true process in (27), which is driven by the true structural innovations $u_{t}$. Thus we now write

$$
X_{t}=\Lambda X_{t-1}+\Gamma u_{t}
$$

From Corollary 2 the autoregressive matrix $\left(I-\widetilde{\beta} H_{\xi}^{\prime}\right) F_{\xi}$ of the filtering error process $f_{t}$ has at most $r-n$ non-zero eigenvalues. Hence for $F_{c} \neq 0, X_{t}$ will be of dimension $2 r-n$, and we can redefine $V$ and $\Lambda$ such that

$$
\begin{aligned}
{\left[\begin{array}{c}
\xi_{t} \\
f_{t}
\end{array}\right] } & =V X_{t} ; \\
V \Lambda V^{-1} & =\left[\begin{array}{cc}
G & -F_{c} \eta^{\prime} \\
0 & \left(I-\widetilde{\beta} H_{\xi}^{\prime}\right) F_{\xi}
\end{array}\right]
\end{aligned}
$$

where $V$ is now $2 r \times(2 r-n)$ and hence $V^{-1}$ is a generalised inverse. The diagonal elements of $\Lambda$ (which become the reciprocal autoregressive roots of the VARMA representation) are given by the non-zero eigenvalues of $G$ and of $\left(I-\widetilde{\beta} H_{\xi}^{\prime}\right) F_{\xi}$. We redefine $\Gamma$ consistently with $(27)$ such that

$$
\Gamma=V^{-1}\left[\begin{array}{c}
I \\
\left(I-\widetilde{\beta} H_{\xi}^{\prime}\right)
\end{array}\right] F_{u}
$$

We also need to redefine $\Psi^{\prime}$ in (F.2), using (16) to write $c_{t}=\eta^{\prime}\left(\xi_{t}-f_{t}\right)$, giving

$$
\Psi^{\prime}=\left[\begin{array}{ll}
H_{\xi}^{\prime}+H_{c} \eta^{\prime} & -H_{c} \eta^{\prime}
\end{array}\right] V
$$


As a result (F.3) no longer holds, but becomes

$$
\left.\Psi^{\prime} \Gamma=\left[\begin{array}{ll}
H_{\xi}^{\prime}+H_{c} \eta^{\prime} & -H_{c} \eta^{\prime}
\end{array}\right]\left[\begin{array}{c}
I \\
\left(I-\widetilde{\beta} H_{\xi}^{\prime}\right.
\end{array}\right)\right] F_{u}
$$

reflecting the impact of structural shocks on both states and filtering errors.

Note that in the restricted case that the states are dynamically exogenous $\left(F_{c}=\right.$ 0 ) by inspection of (F.12) the true states are block recursive (there is no "contamination" of state dynamics by filtering errors), hence the minimal state vector of the true process remains of dimension $r$, as for the observable process.

Given the redefinitions above we can proceed as in the proof of part (a), which allows us to derive a representation of the form ${ }^{7}$

$$
\lambda(L) i_{t}=\left[\operatorname{det}\left[I-\Lambda_{22} L\right] \Gamma+\Phi_{1} \Lambda_{22}^{*}(L) \Gamma_{2} L\right] u_{t}
$$

where now for the general case with $F_{c} \neq 0$, the autoregressive polynomial $\lambda(L)$ is of order $2(r-n)+1$ The right-hand side defines a vector moving average process of order $2(r-n)$, of dimension $n$, in terms of an underlying vector of innovations $u_{t}$ of dimension $s \geq n$

The proof of part (c) of the proposition is now straightforward. A non-fundamental VARMA representation takes the general form

$$
\lambda(L) i_{t}=\theta^{n f}(L) \eta_{t}
$$

where $\eta_{t}$ is an $n$-vector of innovations that cannot be recovered from the history of $i_{t}$ (i.e. $\theta^{n f}(L)$ is non-invertible in non-negative powers of $L$ ), and $\lambda(L)$ and $\theta^{n f}(L)$ are both finite order polynomials. For the case where $n=s=\operatorname{rank}\left(H^{\prime} F_{u}\right)$, it follows immediately, by comparison of (F.16) and (F.17), that $u_{t}$ is (up to a scaling factor) the vector of innovations to a non-fundamental VARMA representation.

By substitution from (F.17) into (F.16) we have, for any nonfundamental representation of the same moving average order ${ }^{8}$

$$
\theta^{n f}(L) \eta_{t}=\left[\operatorname{det}\left[I-\Lambda_{22} L\right] \Gamma+\Phi_{1} \Lambda_{22}^{*}(L) \Gamma_{2} L\right] u_{t}
$$

\footnotetext{
${ }^{7}$ Which implicitly defines $A(L)$ in the exposition in the main text.

${ }^{8}$ Note that even if we fix the moving average order there are still in general multiple (but a finite number of) non-fundamental representations, that can be generated by sequentially replacing a single MA root with its reciprocal, but in which at least one such root is less than unity in absolute value (the representation with all roots greater than unity in absolute value is the unique fundamental representation).
} 
For the case in part (c) where $n=s$, this simply defines a particular $\eta_{t}$ and $\theta^{n f}(L)$. For the general case with $n<s$, inversion of $\theta^{n f}(L)$ involves terms in powers of $F=L^{-1}$, the forward shift operator. Hence non-fundamental innovations can only be recovered from an information set consisting of both the history and future of $u_{t}$, thus proving part (d) of the proposition.

Following Lippi and Reichlin's (1994) terminology, if the representation is of higher autoregressive moving average order than the fundamental representation it is a "nonbasic" representation. By comparison of the order of the representations in (F.7) and (F.16) it is evident that for the general case (i.e. with $F_{c} \neq$ $0)$ the true process results in a $\operatorname{VARMA}(n, 2(r-n)+1,2(r-n))$ representation, hence there is an associated set of non-fundamental representations of the same order, defined by (F.18). By comparison the fundamental representation in (F.7) is $\operatorname{VARMA}(n, r-n+1, r-n)$; hence the non-fundamental representations defined by (F.18) are also non-basic, proving part (e) of the proposition. 
Table 1. Time Series Properties of Observables

\begin{tabular}{|c|c|c|c|c|c|c|c|}
\hline \multirow[t]{3}{*}{ Nature of Information Set } & \multicolumn{3}{|c|}{$\begin{array}{l}\text { Number of Non-Zero Eigenvalues of State } \\
\text { Representation }\end{array}$} & \multicolumn{4}{|c|}{$\begin{array}{l}\operatorname{VARMA}(n, p, q) \text { order of } \\
\text { observables, } i_{t}(p=\text { autoregressive } \\
\text { order, } q=\text { moving average order })\end{array}$} \\
\hline & \multirow{2}{*}{$\begin{array}{l}\text { Full } \\
\text { Information, } \\
\text { equation (10) }\end{array}$} & \multirow{2}{*}{$\begin{array}{l}\text { True } \\
\text { Process, } \\
\text { equation } \\
(25)\end{array}$} & \multirow{2}{*}{$\begin{array}{l}\text { Estimated } \\
\text { Process, } \\
\text { equation (19) }\end{array}$} & \multicolumn{2}{|c|}{ True Process } & \multicolumn{2}{|c|}{$\begin{array}{l}\text { Observable } \\
\text { Process }\end{array}$} \\
\hline & & & & $p$ & $q$ & $p$ & $q$ \\
\hline Instantaneously Invertible & $r$ & $r$ & $r$ & 1 & 0 & 1 & 0 \\
\hline Asymptotically Invertible & $r$ & $r$ & $r$ & $r-n+1$ & $r-n$ & $r-n+1$ & $r-n$ \\
\hline $\begin{array}{l}\text { Non-invertible, } \\
\text { exogenous state process } \\
\left(F_{c}=0\right)\end{array}$ & $r$ & $r$ & $r$ & $r-n+1$ & $r-n$ & $r-n+1$ & $r-n$ \\
\hline $\begin{array}{l}\text { Non-invertible, } \\
\text { dynamically endogenous } \\
\text { state process }\left(F_{c} \neq 0\right)\end{array}$ & $r$ & $2 r-n$ & $r$ & $\begin{array}{l}2(r- \\
n)+1\end{array}$ & $2(r-n)$ & $r-n+1$ & $r-n$ \\
\hline
\end{tabular}

